Santa Clara University

Scholar Commons

Psychology

College of Arts \& Sciences

$12-2014$

\title{
Focusing on the negative: Cultural differences in expressions of sympathy
}

Birgit Koopmann-Holm

Santa Clara University, bkoopmannholm@scu.edu

Jeanne L. Tsai

Follow this and additional works at: http://scholarcommons.scu.edu/psych

Part of the Psychology Commons

\section{Recommended Citation}

Koopmann-Holm, B. \& Tsai, J. L. (2014). Focusing on the negative: Cultural differences in expressions of sympathy. Journal of Personality and Social Psychology, 107, 1092-1115.

Copyright (c) 2014 American Psychological Association. This article may not exactly replicate the final version published in the APA journal. It is not the copy of record.

http://doi.org/10.1037/a0037684

This Article is brought to you for free and open access by the College of Arts \& Sciences at Scholar Commons. It has been accepted for inclusion in

Psychology by an authorized administrator of Scholar Commons. For more information, please contact rscroggin@scu.edu. 


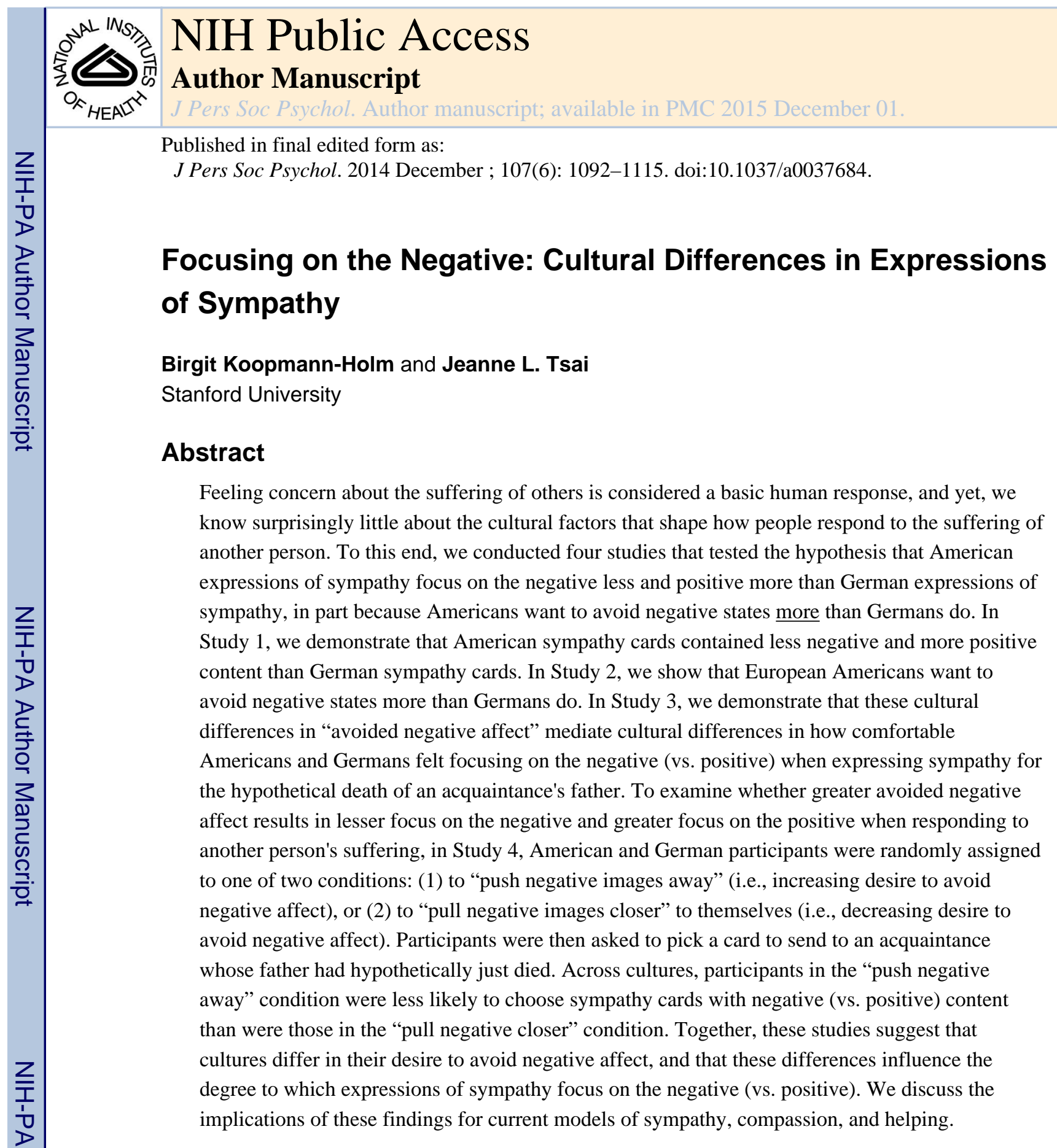

Keywords

Culture; Emotion; Avoided Negative Affect; American; German; Sympathy; Compassion

Imagine that the father of one of your acquaintances just died, and you know she is extremely sad. You want to express your sympathy, so you decide to send her a card. But what type of card do you choose? Do you choose a black and white card that contains

Contact information: Birgit Koopmann-Holm or Jeanne L. Tsai Department of Psychology Bldg. 420, Jordan Hall Stanford University Stanford, CA 94305-2130 birgitkholm@gmail.com or jeanne.tsai@stanford.edu (408) 966-8652 or (650) 736-1843. 
phrases such as "In deep sadness," and "Words will not lighten a heavy heart," or a colorful card that contains phrases such as "Love lives on," and "Memories will bring comfort"?

In this paper, we test the hypothesis that cultural factors shape how people express concern for another person's suffering. Specifically, we predict that because American culture encourages people to avoid negative emotion more than German culture does, American expressions of sympathy focus on the negative less and the positive more than German expressions of sympathy. We conducted four studies to test this hypothesis. Prior to describing the studies, we briefly review previous work on sympathy, compassion, and other feelings of concern about another person's suffering, and then propose how culture may influence expressions of sympathy based on our theoretical framework, Affect Valuation Theory (Tsai, 2007).

\section{Previous Research}

For decades, social scientists have been interested in the functions of sympathy and compassion in everyday life ${ }^{1}$ (e.g., Clark, 1997) as well as the factors that influence these emotions (e.g., Batson et al., 1989; Batson et al., 1991; Cialdini, Darby, \& Vincent, 1973; Davis, 1983; Eisenberg et al., 1989; Eisenberg et al., 1998; Eisenberg \& Miller, 1987; Lennon \& Eisenberg, 1987; Toi \& Batson, 1982). For instance, the empathy-altruism hypothesis originally proposed by Batson and colleagues (Batson, 1981, 1983; Batson et al., 1991) argues that the more people feel distressed (vs. empathetic) when they see another person suffering, the less likely they are to help that person. In contrast, the negative state relief model proposed by Cialdini and colleagues $(1973 ; 1987)$ argues that the more empathetic people feel, the sadder they feel, and people's desire to reduce this sadness leads them to help others. In recent years, there has been a resurgence of interest in sympathy, compassion, and other feelings of concern for another's suffering (e.g., Condon \& DeSteno, 2011; Condon \& Feldman Barrett, 2013; Goetz, Keltner, \& Simon-Thomas, 2010; Keltner, 2009; Nussbaum, 1996; Oveis, Horberg, \& Keltner, 2010; Valdesolo \& DeSteno, 2011; Van Kleef et al., 2008; Zaki \& Ochsner, 2012). However, because the vast majority of these studies have focused primarily on European American samples, we still know little about the role culture plays in shaping people's responses to another's suffering (Wuthnow, 2012, p. 306), despite arguments that the rules and logic surrounding when to give and receive sympathy are culturally shaped (Clark, 1997).

\section{The Role of Culture}

Of the studies that have examined the role of culture, both cultural similarities and differences have been observed. Across different cultures, sympathy and compassion are viewed as emotions (Shaver, Murdaya, \& Fraley, 2001) that can be differentiated from other emotions via touch (Hertenstein, Keltner, App, Bulleit, \& Jaskolka, 2006), have similar triggers (e.g., person who is not responsible for his/her suffering) (Zhang, Xia, \& Li, 2007), and are highly valued (Miller \& Bersoff, 1994). Furthermore, cultural similarities have been observed in the links between experiencing sympathy/compassion and helping others (e.g.,

\footnotetext{
${ }^{1}$ Although scholars differ in their use of the terms "sympathy" and "compassion" to refer to concern for another person's suffering, for the purposes of this paper, we use the terms interchangeably.
} 
helping someone who has just lost a valued toy, or giving money to a needy stranger) (Eisenberg, Zhou, \& Koller, 2001; Kitayama \& Markus, 2000; Trommsdorff, Friedlmeier, \& Mayer, 2007) as well as in the specific patterns of brain activity that occur during empathy for another person's pain (e.g., de Greck et al., 2012; Jiang, Varnum, Hou, \& Han, 2014).

Differences have been observed as well. For instance, on average, people from countries such as Brazil and Costa Rica that value "simpatia" (being concerned about another's wellbeing) and poorer nations such as Malawi and India are more likely to help a blind person, a person with a hurt leg, or someone who has dropped a pen (Levine, Norenzayan, \& Philbrick, 2001) compared to people from countries that do not have a tradition of "simpatia" such as Singapore and Malaysia and wealthier nations such as the Netherlands and the U.S. Indeed, these findings are consistent with work by Stellar, Manzo, Kraus, and Keltner (2012) demonstrating that within the United States, individuals from lower socioeconomic contexts show more compassion towards a peer undergoing a stressful job interview than do those from higher socioeconomic contexts. Other studies suggest that although there are cultural similarities in the patterns of brain activity associated with empathizing with another person's pain, there are also differences (e.g., de Greck et al., 2012; Jiang et al., 2014). For example, whereas Chinese participants showed a pattern of brain activity suggesting that they were regulating their emotions when empathizing with a familiar angry target, German participants showed a pattern of brain activity suggesting that they were assuming the perspective of the angry target (de Greck et al., 2012).

No studies, however, have examined how culture shapes the different ways in which sympathy, compassion, or other feelings of concern for another's suffering might be expressed. Previous research suggests that sympathy and compassion are mixed (i.e., positive and negative) feeling states (e.g., Condon \& Feldman Barrett, 2013; Eisenberg et al., 1994); however, cultures might differ in whether expressions of sympathy focus on the positive (e.g., the "silver lining") or the negative (e.g., the pain and suffering of another). For instance, while most people want to avoid feeling negative, cultures and individuals may differ in the degree to which they want to avoid negative states, which may influence how people express their sympathy. In cultures that encourage people to avoid negative states more, people may focus more on the positive and less on the negative when expressing their sympathy, whereas in cultures that encourage people to avoid negative states less, people may focus on the negative more and positive less. We test this prediction in this paper. First, we define "avoided affect" and then describe the theoretical framework motivating this work, Affect Valuation Theory (Tsai, 2007).

\section{The Role of Avoided Negative Affect}

"Verweinen lasst die Nächte mich, Solang ich weinen mag" [Let me pass the nights in tears, As long as I want to cry] (Johann Wolfgang von Goethe; 1749-1832)

"Be still, sad heart! And cease repining; Behind the clouds is the sun still shining" (Henry Wadsworth Longfellow; 1807-1882)

Feeling negative--- sad, angry, afraid, sluggish----is a part of daily human life. We are passed up for a job; we argue with a friend; we lose a loved one. However, as suggested by 
the above two quotes, people differ in their responses to feeling bad. Whereas some people appear to accept and embrace their negative feelings, as reflected in the quote by German poet Goethe, others try to stop their negative emotions and focus on the positive, as reflected in the quote by American poet Longfellow. Although much research has focused on the experience of negative emotions (e.g., Brainerd, Stein, Silveira, Rohenkohl, \& Reyna, 2008; Chorpita, Albano, \& Barlow, 1998; Forgas, 2008; Frasure-Smith, Lesperance, \& Talajic, 1995; Gross et al., 1997; Gross \& Levenson, 1997; Kiefer, 2005; Kuppens, Realo, \& Diener, 2008), surprisingly few studies have examined variation in the degree to which people want to avoid negative emotions, and the factors that account for such variation.

Instead, studies have demonstrated individual, situational, and cultural differences in people's attitudes toward negative emotion (e.g., Andrade \& Cohen, 2007; Chambless, Caputo, Bright, \& Gallagher, 1984; Grossmann \& Kross, 2010; Harmon-Jones, HarmonJones, Amodio, \& Gable, 2011; Izard, 1971; Sommers, 1984; Tamir \& Ford, 2009; Tamir, Mitchell, \& Gross, 2008; Taylor \& Rachman, 1991; Västfjäll \& Gärling, 2006; Williams, Chambless, \& Ahrens, 1997). However, these studies primarily focus on how much people dislike negative emotions rather than how much people want to avoid negative emotions. For example, Harmon-Jones et al. (2011) examined how much people like or dislike negative emotions (e.g., "I like thinking about sad things", "I dislike how it feels when I am angry"). Similarly, Izard (1971) and Sommers (1984) asked their participants to report how much they dreaded different negative emotions (e.g., "which emotion do you dread the most"; "which emotions do you dread having"), which could reflect both how much people dislike feeling different negative emotions as well as how much they want to avoid feeling negative emotions. Previous research illustrates the differences between liking and wanting (e.g., Dai, Brendl, \& Ariely, 2010), differences that extend to disliking and avoiding as well. For example, while someone might not like feeling sad, she might still want to feel sad (i.e., not want to avoid it) because she believes that feeling sad will allow her to come to a deeper understanding of herself. Although disliking negative emotions and wanting to avoid negative emotions are both important topics of study, we were interested in the latter because we thought that it would influence how people respond to another's suffering. Although some scholars have examined the avoidance of emotions in general (Hayes et al., 2004; Maio \& Esses, 2001), none have examined the desire to avoid negative emotions specifically.

\section{Affect Valuation Theory}

In Affect Valuation Theory (AVT; Tsai, 2007; Tsai, Knutson, \& Fung, 2006), we proposed that: (1) the affective states that people actually feel (their "actual affect") is different from the affective states that people ideally want to feel (their "ideal affect"), (2) cultural factors shape ideal affect more than actual affect, and temperamental factors shape actual affect more than ideal affect, and (3) ideal affect shapes what people do to feel good, as well as their conceptions of health and happiness. Because most people want to feel good, research on ideal affect has primarily focused on positive states (Tsai, 2007; Tsai et al., 2006; Tsai, Louie, Chen, \& Uchida, 2007; Tsai, Miao, \& Seppala, 2007; Tsai, Miao, Seppala, Fung, \& Yeung, 2007). However, here we were interested in the negative states that people want to avoid, and therefore, we briefly discuss how AVT may apply to "avoided affect." 2 
The affective states that people want to avoid feeling (their "avoided affect") should differ from their actual and ideal affect. Whereas ideal affect is a desired state, one that people consciously or unconsciously actively work to achieve, avoided affect is an undesired state, one that people consciously or unconsciously actively work to stay away from or elude. Both are different from actual affect, which is a response to an event or an outcome. In the same way that on average, people do not feel how they want to feel, reflecting a mismatch between actual and ideal affect (e.g., having less fun at a party than one wished), we predict that people may often feel how they do not want to feel, reflecting a mismatch between actual and avoided affect (e.g., getting angry at a friend or loved one).

We also predict that cultural factors shape avoided affect more than actual affect. Rozin (2003) and Shweder (2003) argue that cultural factors shape what people view as good, moral, and virtuous. In AVT, we applied this idea to affective states; i.e., cultural factors shape what affective states people view as good, moral, and virtuous. Similarly, culture should shape the affective states that people view as bad, immoral, and sinful. Indeed, previous research has documented cultural differences in undesirable states (Eid \& Diener, 2001; Izard, 1971; Sommers, 1984). For example, Eid and Diener (2001) found that people in individualistic cultures viewed "guilt" as more undesirable than people in collectivistic cultures. Although cultural factors also shape what affective states people actually feel (Kitayama, Markus, \& Kurokawa, 2000; Mesquita \& Markus, 2004), decades of empirical research suggest that across cultures, actual affect is also shaped by people's temperament (Costa \& McCrae, 1980; David, Green, Martin, \& Suls, 1997; Diener \& Lucas, 1999; Gross, Sutton, \& Ketelaar, 1998; Lykken \& Tellegen, 1996; McCrae, Costa, \& Yik, 1996; Rusting \& Larsen, 1997; Schimmack, Radhakrishnan, Oishi, Dzokoto, \& Ahadi, 2002) as well as their immediate circumstances and regulatory abilities (e.g., Gross, 1998). Thus, we predict that cultural factors should shape avoided affect more than actual affect.

Finally, AVT predicts that avoided affect should influence how people respond to various negative events, including another person's suffering, above and beyond actual affect. If people want to avoid negative states to a great extent, they likely try to avoid people, places, and events that elicit negative emotion (e.g., by trying not to run into an irritable colleague, by not walking home late at night, or by deciding not to see a movie with disturbing content). These attempts, however, may be unsuccessful (e.g., people cannot avoid the irritable colleague, late nights in the office, or unexpected content of films). Under those circumstances, avoided affect should predict how people respond to and cope with the experience of negative emotion. For instance, in response to the death of a loved one, the more individuals want to avoid negative affect, the less they may focus on the negative and the more they may focus on the "silver lining," or the positive aspects of a negative event (e.g., by thinking of the beautiful memories they have of their loved ones) and try to "cease repining" (e.g., by engaging in pleasant activities and encouraging others to do the same). Conversely, the less people want to avoid feeling negative, the less they may focus on the positive and the more they might focus on the negative (e.g., by acknowledging how painful

${ }^{2}$ Of course, there may be specific situations in which avoided affect includes positive states. For example, people might try to avoid excitement initially about an opportunity they have received in order to not be disappointed later. But we propose (and observe) that in general, people want to avoid negative more than positive affective states. 
it is to lose a loved one) and try to "pass the night in tears" (e.g., by allowing oneself to grieve and encouraging others to do the same). We predict that these different responses to and expressions of concern for others' suffering are driven by how much people want to avoid negative affect above and beyond how much they actually experience negative affect (as suggested by some models of helping). The present study is the first to test these predictions, and in doing so, to expand AVT to negative states.

\section{American vs. German Cultural Contexts}

Most research on emotion in cultural and cross-cultural psychology, including our own previous work, has focused on differences between East Asian and Western cultures, and has attributed observed differences to individualism-collectivism. Much less work has focused on variation across different individualistic Western cultures (e.g., American vs. German). The few studies that have do find important variation; for instance, American and German cultures differ in their value priorities: while Americans give higher priority to "mastery" values such as achievement and controlling the environment than Western Europeans (including Germans), Western Europeans give higher priority to "harmony" values such as fitting into the environment than Americans (Schwartz \& Ros, 1995). Furthermore, American and German cultures differ in their display rules of negative emotions (Koopmann-Holm \& Matsumoto, 2011). For instance, the expression of anger and sadness is more acceptable in German than in American contexts. We were particularly interested in comparing Americans and Germans because ethnographic, historical, and personal accounts suggest that Americans want to avoid negative emotions more than Germans do. For instance, German scholar Hedderich (1999) conducted semi-structured interviews with American and German employees (from four American and four German companies), who had spent at least six months in the other country. He asked them about differences between the cultures and concluded that Americans resist talking about their failures, indirectly referring to them as 'items for improvement' (Hedderich, 1999, p. 161) and instead, praise each other for their achievements. Consistent with this observation, in "American Cool," historian Peter Stearns (1994) states that in American society, "fear and anger had no positive function [...]; rather than being directed, they were to be avoided as fully as possible" (p. 96). Indeed, American culture considers "negativity, complaining, pessimism" sinful (Held \& Bohart, 2002, p. 961).

Similarly, McAdams (2004) describes the key feature of contemporary American identity as "the transformation of personal suffering into positive-affective life scenes that serve to redeem and justify one's life" (p. 96), as reflected in American stories, which characteristically have positive endings. Positive endings are also found in American personal accounts of trial and tribulation. For instance, "Chicken Soup for the Soul: The Cancer Book" contains "101 Stories of Courage, Support and Love" (Canfield, Hansen, \& Tabatsky, 2009), implying that even in the face of a life-threatening illness such as cancer, Americans can and should focus on the positive (Ehrenreich, 2009). This may be why some cancer survivors, including cyclist Lance Armstrong, refer to cancer as "the best thing that ever happened" to them (Henneberger, 2013). Indeed, as described in her book, "BrightSided: How positivity thinking is undermining America," when American Ehrenreich (2009) 
publically expressed her anger and anxiety about having breast cancer, other breast cancer patients admonished her and suggested she seek psychological help.

In contrast, Germans openly and explicitly state when something is bad; for instance, many of the respondents in Hedderich's study (1999) commented that Germans are more likely to use the phrase, "das war Mist," or "that was rubbish," when something bad happened, compared to Americans. Unlike American personal accounts of illness, German accounts primarily focus on the negative. For instance, in his book about his battle with cancer entitled “Ein Jahr Hölle [One year of hell], ” German actor Michael Lesch primarily described the anxiety and horror of having cancer, and even years later, talked about cancer as mainly being a horrible experience (Lesch, 2008). Indeed, German culture is often described as being melancholic and pessimistic, as the terms "Weltschmerz" and "Angst" suggest (Clair, 2005; Gelfert, 2005) and as reflected by the "Sturm und Drang" ["Storm and Drive"] movement in German literature and music in the $18^{\text {th }}$ century, in which negative emotions were not just accepted, but glorified.

Why might American and German contexts differ in their desire to avoid negative affect? One possible explanation is that these differences stem from the historical origins of the United States. Although ancestors from both countries originated in Europe, the founding fathers of the United States left Europe to escape religious persecution, famine, and financial hardship (Brooks, 2004; Wilson, 2008). In essence, these individuals responded to their negative life circumstances by dreaming of a "brighter future" in the New World, and by leaving their European homelands to realize that dream (Brooks, 2004; Wilson, 2008). According to the "voluntary settlement hypothesis" (e.g., Kitayama, Ishii, Imada, Takemura, \& Ramaswamy, 2006; Kitayama, Park, Sevincer, Karasawa, \& Uskul, 2009), the traits and values of these early settlers became the basis of American independence and individualism (Lipset, 1997; Wilson, 2008). Several scholars have referred to these traits as the "frontier spirit" (“American Frontier” Spirit; Turner, 1921), which included not only a desire to escape one's negative circumstances to achieve one's dreams but also a willingness to tame and master the wilderness of the New World in order to do so (Conway, Houck, \& Gornick, 2014). In his book, “The Frontier Spirit and Progress," historian Tucker (1980) defined frontiers as "those areas in which men are most dynamically in motion to master the environment" (p. 4).

Thus, by leaving Europe and moving to the United States, the early settlers of the United States may have created a culture in which individuals want to avoid the negative and seek the positive. In contrast, the ancestors of modern Germany (and other parts of Europe) chose to stay in their European homelands. Although they may have stayed for many reasons, one possibility is that instead of dreaming of a new life, they responded to economic and religious hardship by accepting and adjusting to their negative life circumstances. Moreover, they may have preferred their current---albeit imperfect---life circumstances to the idea of crossing a vast ocean and then taming the wilderness of the New World. Thus, these individuals may have been part of or may have created a culture in which individuals are more accepting of the negative, and therefore, have less of a desire to avoid negative emotion. Indeed, a frontier spirit might explain why there are few differences between the US and Germany on the broad dimensions of individualism-collectivism but many 
differences in specific "individualistic" values such as self-enhancement (e.g., achievement) and specific "collectivistic" values such as self-transcendence (e.g., fitting into nature) (e.g., Koopmann-Holm \& Matsumoto, 2011; Schwartz \& Ros, 1995).

In sum, we predicted that contemporary American culture wants to avoid negative states more than contemporary German culture in part because American culture endorses a frontier spirit (i.e., achieving one's goals, influencing one's circumstances, overcoming nature) more than German culture does. Although not the main focus of this paper, we begin to test this hypothesis in Study 3. Many sociologists and historians have written about the frontier spirit and its links to independence and individualism (e.g., Lipset, 1997; Tucker, 1980; Turner, 1921; Wilson, 2008). Additionally, empirical studies have tested these ideas (e.g., Kitayama et al., 2006; Kitayama et al., 2009; Varnum, 2013, 2014; Varnum \& Kitayama, 2011). For instance, one empirical study found that positive emotions were more independent and less interdependent among Japanese living in the frontier of Hokkaido compared to those living in mainland Japan (Kitayama et al., 2006). In the present study, we examine whether differences in the endorsement of specific values associated with the frontier spirit (achievement and fitting into nature) are related to differences in views of negative emotion between the United States and Germany. By comparing views of negative emotion in American and German contexts, this work significantly broadens the current literature on emotion across cultures, which has primarily compared North American and East Asian contexts.

\section{Overview of Studies}

The present studies examined whether cultural differences in expressions of sympathy exist, and if they do, whether cultural differences in the desire to avoid negative affect can explain these differences. Study 1 examined whether American sympathy cards focus less on the negative and more on the positive compared to German sympathy cards. Study 2 examined whether avoided affect is distinct from actual affect and ideal affect, and then examined whether Americans want to avoid negative affect more than do Germans. As described by circumplex/dimensional models of emotion, negative states vary in terms of arousal (Russell, 1980; Russell, Lewicka, \& Niit, 1989). Therefore, in Study 2, we sampled high arousal negative (e.g., fearful, hostile, nervous), low arousal negative (e.g., dull, sleepy, sluggish), and moderate arousal negative (e.g., sad, unhappy, lonely) states to examine whether differences in actual, ideal, and avoided affect held for different types of negative states. Study 3 then examined whether these cultural differences in avoided negative affect mediated cultural differences in expressions of sympathy among Americans and Germans in a hypothetical scenario. In Study 3, we also examined whether cultural differences in avoided negative affect were due to cultural differences in specific frontier values. Finally, in Study 4, we experimentally manipulated avoided negative affect in American and German samples to assess whether we could alter the degree to which people focused on the negative (vs. positive) when expressing sympathy for another's suffering in a hypothetical scenario. 


\section{Study 1: Cultural Differences in Emotional Content of Popular Sympathy Cards}

To begin to examine whether Americans and Germans differ in how they respond to the suffering of another person, we compared the content of American and German sympathy cards. Various studies have examined the content of "cultural products" (e.g., newspapers, books, television, advertisements, architecture, laws, websites), or widely-distributed objects that both reflect and reinforce cultural ideas (e.g., Han \& Shavitt, 1994; Lamoreaux \& Morling, 2012; Markus, Uchida, Omoregie, Townsend, \& Kitayama, 2006; Morling \& Lamoreaux, 2008; Tsai, Louie, et al., 2007; Tsai, Miao, \& Seppala, 2007). Indeed, Morling and Lamoreaux (2008) argue that in some cases, examining the content of widely distributed products is an even better way of illustrating dominant ideas and practices because these products are typically marketed to a general public, and therefore, target culturally shared ideas. This is in contrast to responses to questionnaire measures, for example, which typically reflect a combination of shared (e.g., culture) and unique (e.g., temperament) influences.

Moreover, because these cultural products are widely distributed, members of a culture learn how to think and feel by being exposed to them. For example, in previous work examining cultural differences in ideal affect, our lab compared the emotional content of best-selling American and Taiwanese children's storybooks and observed that American storybooks contained characters with more excited and fewer calm smiles. We then showed that being exposed to storybooks that promoted either excitement or calm altered children's affective preferences assessed immediately after (Tsai, Louie, et al., 2007).

In the present study, we focused on sympathy cards because in American and German contexts, sympathy cards are designed to show one's sympathy and compassion toward someone who has lost a loved one, and to help the receiver cope with his or her pain. In both cultures, the cards are widely distributed and therefore readily available. Moreover, Americans and Germans are regularly exposed to these cards because they are sold in a variety of stores. Finally, because cards are a consumer product like storybooks and magazines, we assumed that when people purchase the cards, they are expressing a preference for the type of emotions conveyed in the cards, which we predict is shaped at least partially by one's cultural values.

In addition to examining American and German sympathy cards, as a control comparison, we compared the emotional content of American and German "baby cards," or cards sent when a friend or acquaintance has just had a baby. We chose baby cards as a control comparison because like sympathy cards, baby cards are sent in response to a significant emotional event in someone's life. However, compared to the death of a loved one, the birth of a baby is typically viewed as a positive event, and therefore, we predicted that cards sent in response to the birth of a baby should not be as influenced by the degree to which a culture encourages people to avoid feeling negative as cards sent in response to the death of a loved one. 


\section{Hypotheses}

We predicted that: (1) American sympathy cards would contain less negative (i.e., fewer words related to sadness and death, lesser acknowledgment of suffering and grief, less dying imagery) and more positive (i.e., more words related to positive feelings and optimism, more encouragement, and more living imagery) content than would German sympathy cards, and (2) cultural differences in the emotional content of sympathy cards would be greater than that of baby cards.

\section{Method}

Card Selection-In order to select a representative sample of sympathy and baby cards that are widely distributed in American and German contexts, we first asked 28 European American $(67.86 \%$ female; mean age $=19.00, \mathrm{SD}=1.12)$ and 22 German undergraduate students $(81.82 \%$ female; mean age $=21.95, \mathrm{SD}=1.53)$ from large top-tier universities in the US and Germany to list the top three places they would go to purchase a sympathy card if they wanted to send one to a friend or acquaintance who had just lost a loved one. We determined how often each store was mentioned as well as whether the store was mentioned first, second, or third (for more information, please contact the first author). European American participants most often mentioned Hallmark, CVS, Target, and Walmart (Target and Walmart were tied). German participants most often mentioned Kaufhof, "Buchhandlung" (German for "bookstore"), and "Schreibwarenladen" (German for "stationary store").

Most of the American cards that the American stores carried were sold by Hallmark and American Greetings (including its greeting card brands, Carlton Cards and Papyrus), and most of the German cards that the German stores carried were sold by bsb, Taurus, Hallmark, and Hanra. We first contacted these companies for a complete catalogue of their cards. In Germany, bsb and Taurus provided their entire catalogues; Hallmark provided a random selection of their cards (cards in their on-line catalogue were not sold in stores), and Hanra provided some cards and suggested that we obtained the others from their website (cards in their on-line catalogue were the same as those sold in stores). This process produced a total of 376 German (246 sympathy, 130 baby) cards.

In the US, Hallmark and American Greetings said that they did not have their entire catalogues and suggested that we purchase the cards at various stores. Therefore, we went to a total of five stores (2 Hallmark, 2 Target, 2 CVS Pharmacy, and 1 Walmart) in California and purchased all the sympathy and baby cards they carried. This process produced 338 American (198 sympathy, 140 baby) cards. ${ }^{3}$

\footnotetext{
${ }^{3}$ In an ideal world, we would have used similar methods to select the cards. Indeed, we had originally tried to obtain the full catalogues of the most popular card companies in the US and Germany. However, while the German card companies provided their full catalogues, the American card companies would not and instead suggested that we go to their stores, which they said contained the full inventory. Although we could have gone to German stores to select the cards, it was less clear whether the German stores had the full inventory of cards (as American stores did). Therefore, we decided it was better to use different approaches to arrive at the same end----obtaining a full inventory of the cards-than to use the same approach to arrive at different ends----a full inventory in one culture, and an incomplete inventory in the other.
} 


\section{Card Coding}

\section{Frequency of negative and positive word use (Percentage of total words that were}

positive or negative): We used the English version of the Linguistic Inquiry and Word Count Program (LIWC; Pennebaker, Francis, \& Booth, 2001) to code the text of the American and German sympathy and baby cards. ${ }^{4}$ A research assistant fluent in English and German translated the content of the German cards into English, and another research assistant also fluent in English and German back-translated these English translations into German to ensure equivalence. Both translators were blind to the study hypotheses. The few discrepancies that arose were discussed, and modifications were made to the original translation to arrive at the best possible English translation of the German text. Then we ran the text of the American and German cards through the English version of LIWC. LIWC calculates the total number of words, and then calculates the percentage of total words that fall into a given category. To assess the frequency of negative words, we added the percentages of total words that fell into the LIWC categories of "Sadness or depression" (e.g., grief, cry, sad) and "Death and dying" (e.g., dead, burial, coffin). To assess the frequency of positive words, we added the percentages of total words that fell into the categories "Positive feelings" (e.g., happy, joy, love) and "Optimism and energy" (certainty, pride, win).

Context of word use: While the types of words that occur in the cards are important, so is the context or way in which each word is used. For instance, the experience of sadness can be encouraged and acknowledged ("take time to grieve") or discouraged ("we hope time will take your grief away"). Therefore, in addition to the LIWC analyses, two trained research assistants (different individuals than those who did the translations-back translations described above), one European American female (who was fluent in English and German and who had lived in the US most of her life) and one German female (who was fluent in German and English and who had lived in Germany most of her life) coded the sympathy cards in their original languages to determine whether the text on the card encouraged/ acknowledged grief $(0=$ no, $1=$ yes $)$ and whether the text on the card encouraged/wished something positive $(0=$ no, 1 = yes). For instance, the phrases "In deep sadness," "Sharing your sorrow," "We mourn the loss" were coded as encouraging/acknowledging grief, and "May you find comfort," "The memories are yours," and "Hold on to hope" were coded as encouraging/wishing something positive. Both coders were blind to study hypotheses. To assess reliability, coders overlapped on $18 \%$ of the cards (79 sympathy cards). Inter-rater reliability was high according to Landis and Koch (1977) (Cohen's kappa for encouraged/ acknowledged grief $=.73$, Cohen's kappa for encouraged/wished something positive $=.74$ ) .

Card Images: The coders (who were the same as the ones who coded the context of word use) also coded the images on the sympathy cards in terms of whether they were living or dying. If there was only one image on the card, that image was coded. If there was more

\footnotetext{
${ }^{4}$ We used the 2001 version of the LIWC internal dictionary because, unlike the 2007 version, the 2001 version includes two positive emotion categories ("positive feelings" and "optimism and energy"). Although these categories were eliminated from the 2007 version because they were rarely used in empirical studies, they are still valid (J. Pennebaker, personal communication, May 29, 2013). These two categories nicely parallel the categories "sadness or depression" and "death and dying," which can be found in both the 2001 and 2007 dictionary version and that are central to our hypotheses.
} 
than one image on the card, the coders coded the most prominent image. Images on the cards were coded as: (1) dying (e.g., shriveled leaves), (2) living (e.g., living flower), or (3) neutral (e.g., rocks, water). Again, inter-rater reliability was high according to Landis and Koch (1977) (Cohen's kappa $=.69)$.

\section{Data Analyses and Results}

We first examined whether the sympathy cards differed in number of words they contained. We conducted a univariate analysis of variance (ANOVA) by Culture (US, Germany) on word count, which we obtained from LIWC. The American sympathy cards contained significantly more words than the German sympathy cards (American mean $=39.10, \mathrm{SE}=$ 1.39; German mean $=10.32, \mathrm{SE}=1.25 ; F[1,442]=237.38, p<.001$, partial eta squared $=$. $35)$. For analyses of frequencies of negative and positive words, we did not control for number of words since these measures were percentage scores, and therefore already took number of words into account. However, for the analyses of context codes (which are based on occurrences), we controlled for the number of words in the cards (our findings did not differ when we did not control for number of words in the cards).

With one exception (for German cards, the correlation between context code of encouraged/ acknowledged grief and LIWC coded sadness/death was .86), the correlations among the variables were generally weak to moderate across cultures, with a mean of $.03(\mathrm{SD}=.17)$ for European Americans and with a mean of $.14(\mathrm{SD}=.38)$ for Germans, suggesting that the codes were non-overlapping. Thus, we concluded that most of the codes referred to different aspects of the cards and therefore should be analyzed separately.

\section{Do American Sympathy Cards Contain Less Negative and More Positive Content Than German Sympathy Cards?}

Frequency of Negative and Positive Words (LIWC) (Percentage of total words that were positive or negative): To examine whether the American and German cards differ in the frequency of negative and positive words (based on LIWC) described above, we conducted a 2 (Culture: American, German) $\times 2$ (Card Type: Sympathy, Baby) $\times 2$ (Valence: negative LIWC category, positive LIWC category) repeated measures ANOVA with Culture and Card Type as between-group factors and Valence as within-group factor. We found a significant main effect for Valence $(F[1,710]=11.14, p=.001$, partial eta squared $=.02)$, which was qualified by significant Valence by Culture $(F[1,710]=10.87, p$ $=.001$, partial eta squared $=.02)$, Valence by Card Type $(F[1,710]=81.32, p<.001$, partial eta squared $=.10)$, and Valence by Culture by Card Type $(F[1,710]=13.42, p<.001$, partial eta squared $=.02$ ) interactions.

As predicted and depicted in Figure 1, simple effects analyses revealed that American sympathy cards contained fewer negative words (American mean $=2.90, \mathrm{SE}=.79$; German mean $=7.30, \mathrm{SE}=.71), F(1,710)=17.35, p<.001$, partial eta squared $=.02)$ and more positive words (American mean $=3.50, \mathrm{SE}=.33$; German mean $=1.35, \mathrm{SE}=.30$ ), $F(1,710$ ) $=23.51, p<.001$, partial eta squared $=.03$, than did German sympathy cards. However, American and German baby cards did not differ in the frequency of negative (American mean $=.04, \mathrm{SE}=.94 ;$ German mean $=.08, \mathrm{SE}=.97 ; F[1,710]=.001, p=.98$, partial eta 
squared $<.001)$ or positive $($ American mean $=5.68, \mathrm{SE}=.39$; German mean $=6.07, \mathrm{SE}=$. $41 ; F[1,710]=.47, p=.50$, partial eta squared $=.001)$ words. ${ }^{5}$

Context of Word Use: Based on the manual coding, we examined whether there were cultural differences in (1) encouraged/acknowledged grief, and (2) encouraged/wished something positive in the sympathy cards. We conducted two logistic regression analyses in which these two variables were regressed onto culture and number of words. As predicted, the American sympathy cards were less likely to encourage/acknowledge grief, $B=-.76, S E$ $=.38, p=.046$, Nagelkerke $\mathrm{R}$ square $=.06$, and were more likely to encourage/wish something positive, $B=2.56, S E=.30, p<.001$, Nagelkerke R square $=.57$, compared to the German cards.

Sympathy Card Images: A chi-square test of independence of the relationship between culture and images revealed that American and German sympathy cards differed in the type of images on the cards, $\chi^{2}[2,444]=50.44, p<.001$, Cramer's $V=.34$. Even though a large percentage of the American (31.31\%) and German sympathy cards (48.78\%) were decorated with images other than dying or living ones, as predicted, significantly fewer American than German cards contained dying images (2.53\% of American cards vs. $16.26 \%$ of German cards). In contrast, more American cards (66.16\%) than German cards (34.96\%) contained living images, $\chi^{2}[1,262]=36.23, p<.001$, Cramer's $V=.37$.

\section{Study 1 Summary and Discussion}

As predicted, American sympathy cards contained less negative and more positive content than German sympathy cards. These cultural differences emerged when we examined the text (both the frequency of negative and positive word use and context in which negative and positive words were used) as well as the images on the cards. Cultural differences in the frequency of positive and negative words (based on LIWC) did not generalize to baby cards, suggesting that they may be specific to primarily negative events, such as the death of a loved one. ${ }^{6}$ While the frequency of positive and negative emotion words in the sympathy cards may appear low, they are well within the range of frequencies reported in the 43 studies listed in the LIWC manual (Pennebaker et al., 2001).

How can these differences in sympathy card content be explained? We hypothesized that these differences are due at least in part to cultural differences in the desire to avoid negative affect. Therefore, in Study 2, we examined whether Americans and Germans indeed differ in the degree to which they want to avoid negative emotion. However, before examining cultural differences in avoided negative affect, we assessed whether avoided negative affect is a separate construct from actual negative affect and ideal negative affect (i.e., we wanted

\footnotetext{
${ }^{5}$ Because the dependent variables were not normally distributed, we conducted Independent-Samples Mann-Whitney U Tests and found similar results.

${ }^{6}$ As mentioned by one reviewer, it is interesting that American and German cultures both respond to having a baby in primarily a positive way, even though some aspects of having a baby are often experienced as negative by parents (e.g., lack of sleep, loss of independence). This may be because when celebrating the birth of a child, both Americans and Germans assume that the parents wished for and chose to have the child, whereas when mourning the death of a loved one, they assume the opposite: i.e., that the mourners did not wish for or choose to lose their loved one. In future work, it would be interesting to examine whether people's responses to positive and negative events varies as a function of the perceived controllability of the event.
} 
to make sure that avoided negative affect is not just the opposite of ideal negative affect), and whether people want to avoid negative states more than they ideally want to or actually feel them, as predicted by Affect Valuation Theory (Tsai, 2007). Because most of our previous work has focused on global actual and ideal affect (i.e., affective states experienced or desired on average or over the course of a typical week), we focused on global avoided affect in this study. We also sampled negative states that varied in terms of high and low arousal, in order to assess whether our findings differed as a function of the type of negative affect.

\section{Study 2: Cultural Differences in Avoided Negative Affect Hypotheses}

We predicted that: (1) avoided negative affect, ideal negative affect, and actual negative affect are distinct factors, and that this distinction holds in American and German contexts, (2) across groups, people want to avoid negative states more than they ideally want to or actually feel them, and (3) European Americans want to avoid feeling negative more than Germans do, controlling for how much they actually experience negative states. ${ }^{7}$

\section{Method}

Participants-One hundred nineteen European American (73.95\% female) students and 104 German students ( $81.73 \%$ female) participated in this study. There were no group differences in gender $\left(\chi^{2}[1,223]=1.93, p=.17\right.$, Cramer's V $\left.=.09\right)$. However, Germans were significantly older (mean age $=23.71, \mathrm{SD}=4.61$ ) than European Americans (mean age $=20.22, \mathrm{SD}=4.47), F(1,221)=32.89, p<.001$, partial eta squared $=.13$. Therefore, we included age in the analyses described below when it emerged as significant covariate. American participants received a \$10 Amazon gift certificate, and German participants received a Euro 7 Amazon gift certificate as compensation for their participation.

\section{Instruments}

Assessment of Actual, Ideal, and Avoided Affect: To assess actual and ideal affect, participants completed the Affect Valuation Index (AVI; Tsai \& Knutson, 2006).

Participants used a five-point scale ranging from 1 ("never") to 5 ("all the time") to rate how often they actually felt and how often they ideally wanted to feel 37 different affective states that sampled each octant of the affective circumplex (high-arousal positive [HAP; elated, enthusiastic, euphoric], positive [P; content, happy, satisfied], low-arousal positive [LAP; peaceful, calm, relaxed], high-arousal negative [HAN; fearful, hostile, nervous], negative [N; sad, unhappy, lonely], low-arousal negative [LAN; dull, sleepy, sluggish], high-arousal [HA; astonished, surprised], and low-arousal [LA; idle, passive, inactive]) (Russell, 1980; Russell et al., 1989) over the course of a typical week. The other affective states sampled were excited, strong, fatigued, angry, activated, rested, quiet, still, contemptuous, guilty, stressed, disgusted, ashamed, and serene ${ }^{8}$. To assess avoided affect, participants used the

\footnotetext{
${ }^{7}$ Throughout the studies presented here, we also assessed the degree to which participants wanted to feel positive states ("ideal positive affect") and actually felt positive states ("actual positive affect"). However, none of the cultural differences in responses to suffering were mediated by ideal or actual positive affect, suggesting again that ideal positive affect and avoided negative affect are distinct constructs.
} 
same rating scale to indicate how often they wanted to avoid feeling the same states over the course of a typical week. Reliabilities are reported below.

Demographic Questionnaire: Participants completed a demographics questionnaire, which assessed participants' gender, age, place of birth, and cultural upbringing.

Procedure-Participants were recruited via email announcements and flyers distributed at large top-tier universities in the US and Germany. European American participants completed all measures in English, and German participants completed all measures in German. Standard translation-back-translation procedures were used. A research assistant fluent in both German and English translated all scales into German. B. K.-H. (also fluent in German and English) then back-translated these German translations into English. The few discrepancies that emerged were discussed and modifications were made to the original translations to arrive at the best possible translation. All participants completed the measures online. The order of the ideal, actual, and avoided versions of the AVI was counterbalanced. Because the order of these measures did not have an effect on the results, we will not discuss it further. Other measures were included in the study as fillers.

\section{Data Analyses and Results}

Given group differences in age, we included age as a covariate. For parsimony, we retained age in the model when it was a significant covariate and removed it when it was not.

\section{Hypothesis 1: Is Avoided Negative Affect Different From Actual and Ideal Negative Affect? ${ }^{9}$-We were first interested in whether among the negative states,} avoided affect was distinct from actual and ideal affect, and if so, whether we would see this differentiation among high arousal negative (HAN), negative $(\mathrm{N})$, and low arousal negative (LAN) states (see above). To test Hypothesis 1, we conducted confirmatory factor analyses using AMOS 21. We included three items from each of the negative octants (HAN: fearful, hostile, nervous; N: sad, unhappy, lonely; and LAN: dull, sleepy, sluggish) for avoided, ideal, and actual affect, and treated each as indicators for the three latent variables of ideal, actual, and avoided negative affect.

We hypothesized a model (see Figure 2) that included nine separate latent variables (avoided HAN, N, and LAN; ideal HAN, N, and LAN; and actual HAN, N, and LAN) and three second-order factors (the first combining avoided HAN, N, and LAN into an overall avoided negative factor, the second combining ideal HAN, N, and LAN into an overall ideal negative factor, and the third combining actual HAN, N, and LAN into an overall actual negative factor). In the model, we allowed these three second-order factors to covary with each other. Thus, our model included 3 covariance links. Across both groups, the confirmatory factor

\footnotetext{
${ }^{8}$ We used "euphoric" rather than "excited" for the HAP composite because the German translation for "excited" is more negative than the English word. Similarly, we used "peaceful" instead of "serene" for the LAP composite because this yielded higher internal consistencies.

${ }^{9}$ To assess whether avoided affect was distinct from other related constructs, participants also completed the general regulatory focus measure, which includes prevention focus (GRFM; Lockwood, Jordan, \& Kunda, 2002), the Attitudes Toward Emotions Scale (ATE; Harmon-Jones et al., 2011) and the Need For Affect Scale (Maio \& Esses, 2001). Correlations between these measures and ours were not significant (for European Americans: -.05 to .17; for Germans: -.11 to .10), demonstrating the discriminant validity of our measure of avoided negative affect.
} 
analysis testing this model revealed that the data fit the model well, $\chi^{2}(312, N=223)=$ $573.34, p<.001$; the root mean square of error approximation (RMSEA) $=.06$, with confidence interval .05 to .07 ; and the Akaike Information Criterion (AIC) $=759.34$. The covariance links between the second-order avoided negative and ideal negative factors (-. $03, p=.049)$, between the second order avoided negative and actual negative factors $(-.02$, $p=.44)$, and between the second order ideal negative and actual negative factors $(.04, p=$. 001) were small to moderate.

To test whether the constructs of ideal and avoided negative affect overlap, we constrained the covariance link between ideal and avoided negative affect to be -1 . Across both groups, this constrained model had a significantly worse fit than the unconstrained model, $\chi^{2}(313, N$ $=223)=775.98, p<.001$; the RMSEA $=.08$, with confidence interval .07 to .09 ; and the $\mathrm{AIC}=959.98, \Delta \chi^{2}=202.64, \Delta \mathrm{df}=1, p<.001$. We also tested whether the constructs of actual and avoided negative affect overlap. To do this, we constrained the covariance link between actual and avoided negative affect to be -1 . Across both groups, this constrained model had a significantly worse fit than the unconstrained model, $\chi^{2}(313, N=223)=$ $725.48, p<.001$; the RMSEA $=.08$, with confidence interval .07 to .08 ; and the AIC $=$ 909.48, $\Delta \chi^{2}=152.14, \Delta \mathrm{df}=1, p<.001$.

In sum, these findings suggest that consistent with Hypothesis 1, avoided negative affect is weakly if at all correlated with actual negative affect and ideal negative affect, and all three types of affect are distinct constructs at both the second-order level (i.e., across all negative states) and at the first-order level (i.e., for each type of negative affect).

Does This Structure Hold Across Groups?-Before we can compare psychological constructs across different groups, we have to demonstrate measurement equivalence (Little, 1997). In order to examine whether the model described above held for European Americans and Germans (i.e., all measurement weights are the same across both groups), we performed a multiple-group confirmatory factor analysis using AMOS 21 (e.g., Cheung \& Rensvold, 1999). All path coefficients were free to vary across the two groups. This unconstrained model had a good fit, $\chi^{2}(624, N=223)=1020.08, p<.001$; the RMSEA $=.05$, with confidence interval .05 to .06 ; and the $\mathrm{AIC}=1392.08$. Then all measurement weights were fixed to be the same across both groups. This fully constrained model provided a significantly worse fit than the unconstrained model, $\chi^{2}(642, N=223)=1056.19, p<.001$; the RMSEA = .05, with confidence interval .05 to .06 ; and the $\mathrm{AIC}=1392.19, \Delta \chi^{2}=36.11$, $\Delta \mathrm{df}=18, p=.01$. Further analysis revealed that the path between the indicator variable ideal hostile and the latent variable ideal HAN varied across cultural groups. When we unconstrained this weight, and fixed all the other measurement weights to be the same across both groups, we found that this constrained model did not provide a significantly worse fit than the unconstrained model, $\chi^{2}(641, N=223)=1046.43, p<.001$; the RMSEA $=.05$, with confidence interval .05 to .06 ; and the $\mathrm{AIC}=1384.43, \Delta \chi^{2}=26.35, \Delta \mathrm{df}=17, p$ $=.07$. This suggests that consistent with Hypothesis 1 , our proposed model holds for European Americans and Germans, except for the link between ideal hostile and ideal HAN affect. Ideal hostile was related to ideal HAN and overall ideal negative affect in the European American (estimate $=1.92, p=.003$ ) but not in the German sample (estimate $=$. $33, p=.06)$. However, the two constructs that we primarily focused on in the present 
studies, avoided negative and actual negative affect, had the same structure for both European Americans and Germans. As we did not find measurement equivalence for the ideal HAN composite, in the subsequent analyses, we only use this composite when we examine the cultural groups separately. Together, these findings suggest that the structure of avoided and actual negative affect is empirically valid and equivalent across American and German contexts.

Mean Aggregates: Correlations and Internal Consistency Estimates-Mean aggregate scores were computed for items that sampled each negative octant of the affective circumplex. For European Americans, Cronbach's alpha's ranged from .64 to .80 for actual negative affect, from .60 to .66 for ideal negative affect, and from .76 to .92 for avoided negative affect. For Germans, it ranged from .69 to .84 for actual negative affect, from .43 to .69 for ideal negative affect, and from .76 to .88 for avoided negative affect.

Calculating mean deviated scores: European Americans had a lower overall mean response to all actual affect items (American mean $=2.47, \mathrm{SE}=.02$; German mean $=2.81$, $\mathrm{SE}=.03 ; F(1,221)=94.70, p<.001$, partial eta squared $=.30)$ and to all ideal affect items (European American mean $=2.44, \mathrm{SE}=.02$; German mean $=2.62, \mathrm{SE}=.02 ; F(1,220)=$ $42.56, p<.001$, partial eta squared $=.16$ ) than did Germans. However, European Americans had a higher overall mean response to all avoided affect items (European American mean $=$ $2.91, \mathrm{SE}=.05 ;$ German mean $=2.54, \mathrm{SE}=.06 ; F(1,219)=22.53, p<.001$, partial eta squared $=.09)$ than did Germans. This is in line with previous observations of AmericanGerman differences in response-style (e.g., Koopmann-Holm \& Matsumoto, 2011).

Because of these cultural differences in response style and previous findings showing that people differ in how much they want to avoid feeling emotions in general (e.g., Maio \& Esses, 2001), we mean deviated participants' responses by subtracting each individual's overall mean response to all the 37 avoided affect items from the raw score for each avoided affect item (e.g., sad), and then calculated the mean aggregate for each type of avoided negative affect (HAN, N, and LAN) (e.g., for avoided HAN, we calculated the mean of mean deviated avoided fearful, avoided hostile, avoided nervous). We followed the same procedure for actual and ideal negative affect. We used these scores for avoided, actual, and ideal negative affect in all subsequent analyses. ${ }^{10}$ Correlations among actual, ideal, and avoided negative affect ranged from $-.35(p<.001)$ to $.64(p<.001)$ for European Americans and from $-.32(p=.001)$ to $.42(p<.001)$ for Germans. ${ }^{11}$

\section{Hypothesis 2: Do People Want To Avoid Negative States More Than They Ideally Want to and Actually Feel Them?-To test Hypothesis 2 that people want to} avoid negative states more than they ideally want to or actually feel them, we conducted pairwise $t$-tests on avoided HAN, N, and LAN with ideal HAN, N, and LAN as well as with

\footnotetext{
${ }^{10}$ In previous work coming from our lab, we ipsatized the AVI scores (i.e., divided the mean deviated scores by the standard deviation across all items). Instead of ipsatization, we chose to use mean deviation in order to be able to use the same scores for all analyses reported in the present paper, including correlational analyses, for which ipsatized scores are not recommended. We also ran our analyses using the raw data, and the results were very similar.

${ }^{11}$ We also examined the correlations between avoided negative (HAN, N, and LAN) and ideal positive (HAP, P, and LAP) affect. For European Americans, they ranged from $r(119)=.01, p=.91$ to $r(119)=.28, p=.002$. For Germans, they ranged from $r(102)=.001, p$ $=.995$ to $r(102)=.14, p=.16$.
} 
actual HAN, N, and LAN affect for Americans and Germans separately. We also compared the means of ideal HAN, N, and LAN with actual HAN, N, and LAN. All means statistically differed from each other, $p<.001$. As Figure 3 shows, European Americans (top graph) and Germans (bottom graph) wanted to avoid HAN, N, and LAN states more than they ideally wanted to feel or actually felt them. These findings support the above findings that actual negative affect, ideal negative affect, and avoided negative affect are distinct constructs. For the rest of the paper, we focus on avoided and actual negative affect; however, we ran all of the analyses controlling for ideal negative affect to ensure that the results were not due to ideal negative affect. The pattern of our findings did not change, and therefore, we do not discuss ideal negative affect further.

\section{Hypothesis 3: Do Americans Want to Avoid Feeling Negative More Than Do Germans?-We initially ran our analyses with Gender as a between subjects factor;} however, because there were no significant main effects or interactions involving Gender, we dropped Gender from our final analyses. We controlled for actual affect when examining group differences in avoided affect, and we controlled for avoided affect when examining group differences in actual affect to determine the independent effects of group on each type of affect. The findings did not change when we excluded these covariates.

To test Hypothesis 3 that European Americans want to avoid feeling negative states more than Germans do, we conducted a $2 \times 2 \times 3$ (Group: European Americans, Germans $\times$ Affect Type: Avoided Affect, Actual Affect $\times$ Octant: HAN, N, LAN) repeated measures analysis of covariance (ANCOVA) with Group as between-subjects factor and Affect Type and Octant as within-subjects factors, controlling for age. The Group by Affect Type interaction was significant, $F(1,218)=14.54, p<.001$, partial eta squared $=.06$ as well as the Group by Affect Type by Octant interaction, $F(1.82,397.65)=5.55, p=.01$, partial eta squared $=.03$. Consequently, for avoided and actual affect, we conducted separate ANCOVAs for avoided and actual negative affect for each of the three different octants. More specifically, we conducted three separate ANCOVAs on avoided HAN, N, and LAN, with Group (European Americans, Germans) as the independent variable, controlling for actual HAN, N, and LAN, respectively, and age when it emerged as a significant covariate. As predicted and depicted in Figure 4 (left), European Americans wanted to avoid HAN, N, and LAN states significantly more than did Germans (HAN: European American mean $=$ $1.31, \mathrm{SE}=.06$; German mean $=.97, \mathrm{SE}=.07, F(1,217)=13.62, p<.001$, partial eta squared $=.06 ; \mathrm{N}$ : European American mean $=1.27, \mathrm{SE}=.07$, German mean $=.91, \mathrm{SE}=.07$, $F(1,218)=12.10, p=.001$, partial eta squared $=.05$; LAN: European American mean $=$ $1.15, \mathrm{SE}=.05$, German mean $=.95, \mathrm{SE}=.06, F(1,219)=5.68, p=.02$, partial eta squared $=.03)$.

We also examined group differences in actual negative affect. We conducted ANCOVAs on actual HAN, N, and LAN separately with group as the independent variable, controlling for avoided HAN, N, and LAN, respectively and age when it emerged as a significant covariate. As depicted in Figure 4 (right), European Americans and Germans did not differ in how much they actually felt HAN (European American mean $=-.46, \mathrm{SE}=.05$; German mean $=$ $-.34, \mathrm{SE}=.05 ; F[1,217]=2.45, p=.12$, partial eta squared $=.01$ ) or LAN (European American mean $=.12, \mathrm{SE}=.06 ;$ German mean $=-.01, \mathrm{SE}=.06 ; F[1,219]=2.35, p=.13$, 
partial eta squared $=.01)$. Although European Americans (marginal mean $=-.23, \mathrm{SE}=.06$ ) actually felt $\mathrm{N}$ states significantly less than did Germans (marginal mean $=-.05, \mathrm{SE}=.06$ ), $F(1,219)=4.07, p=.045$, partial eta squared $=.02$, the effect size of the cultural difference in actual $\mathrm{N}$ was smaller than that of the cultural differences in avoided $\mathrm{N}, \mathrm{HAN}$, and LAN.

\section{Study 2 Summary}

As predicted, our results suggest that (1) avoided negative affect, ideal negative affect, and actual negative affect are distinct constructs, and that this holds for European Americans and Germans, (2) across groups, people want to avoid negative states more than they ideally want to or actually feel them, and (3) European Americans want to avoid feeling negative more than do Germans, even after controlling for actual negative affect. Furthermore, while we found cultural differences for all three negative octants of avoided affect, we only found cultural differences for one out of the three negative octants of actual affect. This suggests that consistent with AVT and our predictions, culture shapes avoided affect more than actual affect.

In Study 1, we established that there are cultural differences in expressions of sympathy, and in Study 2, we demonstrated that there are cultural differences in the desire to avoid negative affect. In Study 3, we examined whether there was a direct link between how much people want to avoid negative affect and the degree to which their expressions of sympathy focused on the negative (vs. positive). In addition, we also examined whether cultural differences in avoided negative affect were due to differences in the endorsement of specific frontier values.

\section{Study 3: Do Cultural Differences in Avoided Negative Affect Mediate Cultural Differences in Expressions of Sympathy?}

In Study 3, participants completed measures of avoided affect, and then were asked to rate and choose between sympathy cards that either focused more on the negative or more on the positive. Because Study 2 revealed that European Americans wanted to avoid HAN, LAN, and $\mathrm{N}$ states more than did Germans, for parsimony, in Study 3, we collapsed across the three types of negative affect and created a general avoided negative affect score. ${ }^{12}$

\section{Hypotheses}

We predicted that: (1) European Americans would report feeling less comfortable sending sympathy cards that focus on the negative and more comfortable sending sympathy cards that focus on the positive compared to Germans, (2) European Americans would report wanting to avoid feeling negative more than would Germans, (3) cultural differences in avoided negative affect would mediate the cultural differences in ratings of negative and positive sympathy cards, and (4) cultural differences in avoided negative affect would be mediated by frontier values (i.e., the more individuals value achievement, influencing their environments, and overcoming nature, the more they would report wanting to avoid negative affect).

\footnotetext{
${ }^{12}$ The pattern of results for each of the negative octants (LAN, N, HAN) was the same.
} 


\section{Method}

Participants-Ninety-seven European American students and 93 German students participated in this two-part online study. European Americans were born, raised, and had lived most of their lives in the US and had parents who were both of European descent. Germans were born, raised, and had lived most of their lives in Germany, and had parents who were both of German descent. The two groups differed in gender distribution (European American: 63.92\% female; German: 82.61\% female, $\chi^{2}[1,189]=8.37, p=.004$, Cramer's V = .21) and age, with the German sample being more female and older (mean age $=24.00, \mathrm{SE}=.33)$ than the European American sample (mean age $=22.18, \mathrm{SE}=.32), F(1$, $186)=15.97, p<.001$, partial eta squared $=.08$. In our initial analyses, we included gender and age as covariates; however, because the findings were similar to when they were not included as covariates, we dropped them from our final analyses. Participants received an \$8/Euro 6 Amazon gift certificate as compensation for completing the first part and another \$12/Euro 10 Amazon gift certificate for completing the second part of the online study.

Procedure-Participants were recruited via email announcements for a two-part "emotions and consumer products study" at large top-tier universities in the US and Germany. All measures of interest were administered in the first part of the survey. European American participants completed all measures in English, and German participants completed all measures in German. The same translation-back-translation procedures as in Study 2 were used to ensure equivalence of the measures. All participants completed the measures online at home.

\section{Instruments}

Actual and Avoided Affect: As in Study 2, to assess actual and avoided affect, participants completed the AVI. As in Study 2, compared to Germans, Americans had a lower overall mean response to all actual affect items (American mean $=2.37, \mathrm{SE}=.02$; German mean $=$ $2.82, \mathrm{SE}=.02 ; F(1,187)=185.50, p<.001$, partial eta squared $=.50)$, but a higher overall mean response to all avoided affect items (American mean $=3.02, \mathrm{SE}=.05$; German mean $=2.78, \mathrm{SE}=.05 ; F(1,186)=10.11, p=.002$, partial eta squared $=.05)$. Therefore, as in Study 2, we mean-deviated actual and avoided negative affect scores. As stated above, for parsimony, we collapsed across the specific types of negative affect (HAN, LAN, N) because group differences emerged for all three type of negative affect in Study 2. Thus, we computed mean aggregate scores for the negative items (sad, unhappy, lonely, fearful, hostile, nervous, dull, sleepy, and sluggish). Internal consistencies (Cronbach's alphas) for actual and avoided negative affect were .77 , and .93 for Americans and .80 , and .90 for Germans, respectively.

Cultural values: Participants completed the 57-item version of the Schwartz Value Survey (SVS; Schwartz, 1992). Participants were presented a list of 57 values; for each value, participants used a 9-point scale ranging from -1 (“opposed to my values") to 7 ("of supreme importance") to indicate how important each value was to them. To derive an overall frontier value score, we first aggregated responses to "successful (achieving goals)," "capable (competent, effective, efficient)," "ambitious (hardworking, aspiring)," "influential (having an impact on people and events)," and "intelligent (logical, thinking)" to derive an 
"achievement value score" based on Schwartz (2012) (Cronbach's alpha $=.74$ for European Americans, .72 for Germans). We then aggregated responses to "unity with nature (fitting into nature)" and "protecting the environment (preserving nature)," to derive a "fitting into nature value score", which we viewed as the opposite of a willingness to overcome and master nature (Conway et al., 2014) (Cronbach's alpha $=.85$ for European Americans, .74 for Germans). Because we wanted to capture how much people value achievement compared to how much they value fitting into nature, we then subtracted the "fitting into nature" value score from the "achievement" value score to create an overall frontier value score. This difference score reflects the relative strength of both values, an approach used by Kitayama et al. (2009) in the context of independence and interdependence.

Rating of Sympathy Cards: To assess expressions of sympathy, we created three pairs of cards based on the American and German sympathy cards in Study 1. Each pair contained one card that focused on the negative more, and one that focused on the positive more. We created cards (available upon request) that could be found in both cultures, and therefore, were more negative and less positive than most American cards and less negative and more positive than most German cards. Participants saw the three pairs successively, first rating each card in the pair and then choosing one of the cards from each pair. For each pair, the text on the cards was matched in length, overall theme (e.g., nature), and card images so that the cards mainly differed in their affective content. For instance, a negative card read "A severe loss... take time to grieve" and a positive card read "Remembering... let time heal your soul." We counterbalanced the order of the cards within each pair (while the order of the pairs themselves always remained the same), and we switched the background images on the covers of the cards within each pair. Because the order of the cards as well as the different background images did not influence our findings, we will not discuss them further.

Participants read the following instruction: "Please imagine that the father of one of your acquaintances just died, and you would like to send a card to this acquaintance. Please look at the cards below and rate how comfortable you would feel sending each card to your acquaintance." Then participants saw one pair of sympathy cards. Participants made their ratings on a 5-point scale ranging from 1 ("extremely uncomfortable") to 5 ("extremely comfortable") immediately after they saw each card in each pair. After participants rated both cards, participants were asked, "If you had to choose between the two cards above, which card would you send to your acquaintance?" Participants did the same for the second and third pairs of cards.

Although we created the cards, participants may have heard the phrases on the cards or seen the images on the cards before. Because familiarity increases liking (Zajonc, 2001), we asked participants to indicate whether or not they had seen any of the images or heard any of the phrases on the cards before. 3.09\% of American and 3.23\% of German participants reported being familiar with all three card pairs, and therefore, we excluded these participants from the analyses (these percentages did not differ between the two countries, $\chi^{2}$ $(1,190)=.003, p=.96$, Cramer's V $=.004)$. 
We calculated the average comfort rating of the three negative cards and the average comfort rating of the three positive cards for each participant. Internal consistencies (Cronbach's alphas) for these aggregates of the three negative and three positive cards were . 29 and .69 for Americans and .59 and .57 for Germans, respectively. The very low value (. 29) for Americans' aggregate for the negative cards reflects the restricted variance of the ratings in the American sample.

Demographic Questionnaire: Finally, participants completed a demographics questionnaire, which asked for participants' gender, age, ethnicity, place of birth and upbringing, as well as their parents' and grandparents' ethnicities. Because religiosity and belief in an afterlife might influence the type of sympathy cards people feel most comfortable sending (e.g., people may be more positive if they think that the deceased is going to heaven), participants also indicated how religious they were on an eight-point scale ranging from 0 ("not at all") to 7 ("very religious") and whether or not they believed in life after death. There were no cultural differences in these variables, and therefore, we do not discuss them further.

\section{Study 3 Analyses and Results}

\section{Hypothesis 1: Do Americans and Germans Differ in Their Rating and Choice of Sympathy Cards?}

To test Hypothesis 1, we conducted a $2 \times 2$ (Group: European Americans, Germans $\times$ Valence: mean rating of negative cards, mean rating of positive cards) repeated measures ANOVA with Group as between-subjects factor and Valence as within-subjects factor. There was a significant main effect of Valence $(F[1,181]=92.40, p<.001$, partial eta squared $=.34)$, but this was qualified by a significant Group $\times$ Valence interaction $(F[1$, $181]=32.86, p<.001$, partial eta squared $=.15)$. As predicted, simple effects analyses revealed that Americans felt less comfortable sending the negative cards (Americans: Mean $=2.05, \mathrm{SE}=.08$; Germans: Mean $=2.64, \mathrm{SE}=.08 ; F[1,181]=25.00, p<.001$, partial eta squared $=.12$ ) and more comfortable sending the positive cards than did Germans (Americans: Mean $=3.22, \mathrm{SE}=.10$; Germans: Mean $=2.94, \mathrm{SE}=.10 ; F[1,181]=4.00, p$ $=.047$, partial eta squared $=.02$ ), as illustrated in Figure 5a. Although both European Americans and Germans felt more comfortable sending the positive than the negative cards, this difference was much larger for European Americans than Germans (European Americans: $F[1,181]=121.04, p<.001$, partial eta squared $=.40$; Germans: $F[1,181]=$ $7.33, p=.01$, partial eta squared $=.04$, respectively).

We also examined whether there were cultural differences in the percentage of participants who chose at least one out of three negative cards. Among Americans, only 36.56\% chose at least one negative card from the three pairs, whereas among the Germans, $72.22 \%$ did, $\chi^{2}(1$, $183)=23.43, p<.001$, Cramer's V $=.36$. 


\section{Hypothesis 2: Do Americans and Germans Differ in How Much They Want To Avoid Feeling Negative?}

To test Hypothesis 2, we conducted a $2 \times 2$ (Group: European Americans, German $\times$ Affect Type: Avoided Affect, Actual Affect) repeated measures ANCOVA with Group as betweensubjects factor and Affect Type as within-subjects factor. The Group by Affect Type interaction was significant, $F(1,181)=13.38, p<.001$, partial eta squared $=.07$. Consequently, we conducted a univariate ANCOVA by Group (European Americans, Germans) for avoided negative affect, controlling for actual negative affect. As predicted, consistent with Study 2, and illustrated in Figure 5b, European Americans wanted to avoid feeling negative more than did Germans (European Americans: Mean $=1.32, \mathrm{SE}=.05$; Germans: Mean $=1.15, \mathrm{SE}=.05 ; F[1,180]=7.18, p=.01$, partial eta squared $=.04$ ).

We also examined group differences in actual negative affect. We conducted a univariate ANCOVA by Group (European Americans, Germans) for actual negative affect, controlling for avoided negative affect. Contrary to prediction, European Americans actually felt negative states less than did Germans (European Americans: Mean $=-.25, \mathrm{SE}=.04$; Germans: Mean $=-.10, \mathrm{SE}=.04 ; F[1,180]=7.38, p=.01$, partial eta squared $=.04)$, and the magnitude of this difference was comparable to that of avoided negative affect.

\section{Hypothesis 3: Does Avoided Negative Affect Mediate Cultural Differences in Expressions of Sympathy?}

To examine whether avoided negative affect mediated the relationship between group and rating of the sympathy cards, we conducted a series of regression analyses as outlined by Baron and Kenny (1986), Kenny, Kashy, and Bolger (1998), and MacKinnon, Lockwood, Hoffman, West, and Sheets (2002). We first examined ratings of sympathy cards that focused on the negative and then ratings of sympathy cards that focused on the positive. The zero-order correlations between the variables in the regressions are depicted in Table 1. Predictors that are not explicitly mentioned below were not significant.

Rating of Negative Sympathy Cards-In Step 1, we regressed the mean rating of the negative sympathy cards, controlling for the mean rating of the positive sympathy cards (to control for overall comfort with sending sympathy cards), onto Group (path c). As already described by the ANCOVA above, Germans felt more comfortable sending the negative cards, $B_{c}=.67, S E=.11, t(180)=5.90, p<.001$, than did Americans. Furthermore, the more comfortable people felt sending the positive cards (covariate), the more comfortable they felt sending the negative cards, $B=.27, S E=.06, t(180)=4.64, p<.001$, Cohen's $\mathrm{f}^{2}=$. 27 for the whole model. In Step 2, we regressed avoided negative affect on Group, controlling for actual negative affect (path a) because of observed cultural differences in actual negative affect. As described above, Americans wanted to avoid feeling negative more than did Germans, $B_{a}=-.17, S E=.07, t(180)=-2.68, p=.01$, Cohen's $\mathrm{f}^{2}=.05$ for the whole model. In the third step, we regressed the mean rating of the negative cards onto avoided negative affect (path b) and Group (path c'). We also entered actual negative affect (to assess the influence of avoided negative affect above and beyond actual negative affect) and the mean rating of the positive cards (to control for overall comfort with cards) as covariates. Above and beyond group differences, the more people wanted to avoid negative 
affect, the less comfortable they were sending the negative cards, $B_{b}=-.27, S E=.13, t(177)$ $=-2.12, p=.04$, Cohen's $\mathrm{f}^{2}=.32$ for the whole model (See Figure 6 ). Also, the more comfortable people felt sending the positive cards (covariate), the more comfortable they felt sending the negative cards, $B=.29, S E=.06, t(177)=4.91, p<.001$. Furthermore, after entering avoided negative affect in the model, the effect of Group on the rating of the negative cards (path c') was significantly reduced, $B_{c^{\prime}}=.60, S E=.12, t(177)=5.16, p<$. 001, difference in coefficients test by Freedman and Schatzkin (MacKinnon et al., 2002): $t_{B c-B c^{\prime}}(180)=3.04, p=.003$. Thus, as predicted, avoided negative affect partly mediated the cultural differences in the rating of the negative cards. Actual negative affect was not a significant predictor in this model, $B=.26, S E=.15, t(177)=1.76, p=.08$, and therefore, it did not mediate differences in expressions of sympathy.

We also tested the indirect effect of group on rating of the negative cards through avoided negative affect, using Preacher and Hayes' (2008) bootstrapping macro with 5,000 resamples. This indirect effect was estimated to lie between .003 and .119 with $95 \%$ confidence interval. Because this $95 \%$ confidence interval does not include zero, the indirect effect is significantly different from zero at $p<.05$. In line with the difference in coefficients test reported above, this suggests that avoided negative affect can partly explain AmericanGerman differences in the comfort rating of the negative cards. ${ }^{13}$

Rating of Positive Sympathy Cards-We also performed a multiple regression analysis on the mean rating of the positive sympathy cards, entering avoided negative affect, actual negative affect, and the mean rating of the negative cards (to control for overall comfort with sending sympathy cards) in one step. Although the comfort rating of the positive sympathy cards was significantly related to actual negative affect, $B=-.58, S E=$. $18, t(178)=-3.29, p=.001$, as well as to the rating of the negative cards, $B=.34, S E=.08$, $t(178)=4.06, p<.001$, it was not related to avoided negative affect, $B=.21, S E=.16$, $t(178)=1.29, p=.20$, Cohen's $\mathrm{f}^{2}=.14$ for the whole model. These findings did not support Hypothesis $3 .{ }^{14}$

\section{Hypothesis 4: Do Frontier Values Mediate Cultural Differences in Avoided Negative Affect?}

To examine whether frontier values mediated the relationship between group and avoided negative affect, we conducted the same series of regression analyses as outlined for the mediational analyses described above.

\footnotetext{
${ }^{13}$ The reverse mediation was not significant.

${ }^{14}$ Actual negative affect mediated the relationship between culture and rating of the positive sympathy cards. Controlling for the mean rating of the negative cards, Germans felt less comfortable sending the positive cards than did European Americans, $B_{c}=-.52, S E=$. $14, t(180)=-3.59, p<.001$, Cohen's $\mathrm{f}^{2}=.14$ for the whole model. Furthermore, controlling for avoided negative affect, Germans actually felt negative more than did Americans, $B_{a}=.16, S E=.06, t(180)=2.72, p=.01$, Cohen's $\mathrm{f}^{2}=.05$ for the whole model. Finally, controlling for avoided negative affect and the mean rating of the negative cards, above and beyond group differences, the more people actually felt negative affect, the less comfortable they were sending the positive cards, $B b=-.49, S E=.18, t(177)=$ $-2.81, p=.01$, Cohen's $f^{2}=.20$ for the whole model. After entering actual negative affect in the model, the effect of Group on the rating of the positive cards was reduced, $B_{C^{\prime}}=-.45, S E=.15, t(177)=-3.11, p=.002$, difference in coefficients test by Freedman and Schatzkin (MacKinnon et al., 2002): $t_{B c}-B c^{\prime}(182)=-2.47, p=.01$. The indirect effect of group on rating of the negative cards through actual negative affect was estimated to lie between -.191 and -.007 with $95 \%$ confidence interval. Thus, Americans felt more comfortable sending the positive cards in part because they actually felt less negative than their German counterparts (but see Study 4 for different results).
} 
In Step 1, we regressed avoided negative affect, controlling for actual negative affect, onto Group (path c). As described above, Americans wanted to avoid feeling negative more than did Germans, $B_{c}=-.17, S E=.07, t(180)=-2.68, p=.01$, Cohen's $\mathrm{f}^{2}=.05$ for the whole model. In Step 2, we regressed frontier values on Group (path a). As predicted, Americans endorsed frontier values more than did Germans, $B_{a}=-.77, S E=.28, t(182)=-2.79, p=$. 01, Cohen's $\mathrm{f}^{2}=.04$. In the third step, we regressed avoided negative affect onto frontier values (path b) and group (path c'). We also entered actual negative affect as control variable. Above and beyond group differences, the more people endorsed frontier values, the more they wanted to avoid feeling negative affect, $B_{b}=.04, S E=.02, t(179)=2.21, p=.03$, Cohen's $\mathrm{f}^{2}=.08$ for the whole model. Furthermore, after entering frontier values in the model, the effect of Group on avoided negative affect (path c') was significantly reduced, $B_{c^{\prime}}=-.14, S E=.07, t(179)=-2.16, p=.03$, difference in coefficients test by Freedman and Schatzkin (MacKinnon et al., 2002): $t_{B c}-B c^{\prime}(181)=-2.13, p=.03$. These findings suggest that as predicted, frontier values partly mediated the cultural differences in avoided negative affect.

We also tested the indirect effect of group on avoided negative affect through frontier values, using Preacher and Hayes' (2008) bootstrapping macro with 5,000 resamples. This indirect effect was estimated to lie between -.079 and -.003 with $95 \%$ confidence interval. Because this $95 \%$ confidence interval does not include zero, the indirect effect is significantly different from zero at $p<.05$. In line with the difference in coefficients test reported above, this suggests that frontier values can partly explain American-German differences in avoided negative affect.

We also performed a multiple regression analysis on actual negative affect, entering frontier values and avoided negative affect in one step. Frontier spirit values were not significantly related to actual negative affect, $B=.02, S E=.02, t(180)=1.00, p=.32$, Cohen's $\mathrm{f}^{2}=.01$ for the whole model. Therefore, we did not pursue the mediational analyses further.

These findings suggest that Americans want to avoid feeling negative emotions more than Germans do in part because they endorse frontier values more than Germans do. ${ }^{15}$

\section{Study 3 Summary}

Consistent with Hypothesis 1, Americans were less comfortable sending sympathy cards that focused on the negative and were more comfortable sending sympathy cards that focused on the positive compared to Germans. Interestingly, both groups felt more comfortable sending the positive cards than the negative cards, although this difference was more pronounced for Americans (effect size $=.40$ ) than Germans (effect size $=.04$ ). In part, this may be because we created cards that could be found in both cultural contexts, and therefore, were more moderate in their positivity and negativity than cards that are actually found in the two cultures.

\footnotetext{
${ }^{15}$ We conducted meditational analyses that treated each component (achievement, fitting into nature) separately, but the meditational analyses were only significant when the difference (achievement minus fitting into nature) was the mediator, suggesting that it is the relative value placed on achievement vs. unity of nature that differentiates the cultures and that accounts for differences in avoided negative affect.
} 
Consistent with Hypothesis 2 and Study 2 findings, Americans wanted to avoid feeling negative states more than did Germans. ${ }^{16}$ Interestingly, unlike Study 2, here we also found cultural differences in actual negative affect that were of similar effect size as the cultural differences in avoided negative affect. In part, this may reflect when we conducted the two studies. We conducted Study 2 in 2007, and Study 3 in 2012. In 2007, both the US and Germany were equally affected by the global financial crisis, whereas in 2012, Germans were more hurt by the European sovereign-debt crisis compared to Americans. Thus, in 2012, the emotional climate was worse in Germany than in the US (Eddy \& Jolly, 2013), which could explain why the group differences in actual negative affect were more pronounced in Study 3 than in Study 2.

Consistent with Hypothesis 3, avoided negative affect partly mediated the cultural differences in the comfort rating of the negative sympathy cards. However, contrary to Hypothesis 3, avoided negative affect was not correlated with comfort ratings of the positive sympathy cards. This suggests that avoided negative affect might be particularly relevant to negative stimuli.

Finally, consistent with Hypothesis 4, cultural differences in the desire to avoid negative affect were partly due to cultural differences in endorsement of specific frontier values (i.e., the relative importance of achievement vs. fitting into nature). While these data lend some support to our hypothesis, future studies are needed to determine the causal direction of this relationship. In addition, studies with larger sample sizes are needed to test the full model, in which frontier values mediate cultural differences in avoided negative affect, which in turn should mediate cultural differences in sympathy card ratings.

Together, our findings from Studies 1-3 suggest that cultural differences in expressions of sympathy are due to cultural differences in the desire to avoid negative affect. One clear limitation of Studies 2 and 3, however, is their correlational design. Consequently, we do not know the causal direction of the relationship between avoided negative affect and comfort ratings of the negative sympathy cards. To examine whether there is a causal relationship, in Studies $4 \mathrm{a}$ and $4 \mathrm{~b}$, we manipulated the degree to which people wanted to avoid negative affect and then examined the type of sympathy card (negative vs. positive) they preferred to send.

\section{Study 4: Does Manipulating Avoided Negative Affect Alter Expressions of Sympathy?}

According to the embodied cognition theory (Anderson, 2003), the role of the physical body (e.g., the way we move) has properties that affect a person's mental processes. Based on this theory, we hypothesized that avoided affect could be manipulated through a physical task. Thus, in Study 4, we manipulated avoided negative affect using an adapted version of the “Approach-Avoidance Task" by Rinck and Becker (2007). In our study, using a joystick, participants were instructed to use a joystick to either push negative images away (to

\footnotetext{
${ }^{16}$ In Study 2 and 3 we found that Germans want to avoid feeling positive emotions more than Americans, arguing against the possibility that Americans are just higher in avoidance motivation.
} 
increase the desire to avoid negative affect) or pull negative images closer (to decrease their desire to avoid negative affect). In Study 4a, we examined whether the manipulation indeed altered reports of their desire to avoid negative affect. In Study $4 \mathrm{~b}$, we examined whether the manipulation altered the choice of negative versus positive sympathy cards. We conducted two separate studies (4a and 4b) on different samples to ensure that the administration of the manipulation check (i.e., the measures of actual and avoided affect) did not interfere with the dependent variable (i.e., choice of sympathy cards).

\section{Study 4a: Manipulation Check \\ Hypotheses}

We predicted that, across groups, participants in the "push negative away" condition would report wanting to avoid negative affect more than those in the "pull negative closer" condition. We also predicted that, across conditions, Americans would report wanting to avoid negative affect more than would Germans.

\section{Method}

Participants-Thirty-two American students (48.39\% European American; 62.50\% female; mean age $=18.97, \mathrm{SE}=.35)$ and 30 German students $(53.33 \%$ female; mean age $=$ $23.57, \mathrm{SE}=.36$ ) were randomly assigned to either the "push negative away" condition (US: $\mathrm{n}=18$; Germany: $\mathrm{n}=15$ ) or the "pull negative closer" condition (US: $\mathrm{n}=14$; Germany: $\mathrm{n}=$ $15)$. The two cultural groups did not differ in gender distribution $\left(\chi^{2}[1,62]=.53, p=.47\right.$, Cramer's V $=.09$ ), but Germans were significantly older than Americans, $F[1,60]=84.54$, $p<.001$, partial eta squared $=.59$. Therefore, we controlled for age in the analyses below when it emerged as significant covariate.

\section{Task and Instruments}

Joystick Task: We adapted the "Approach-Avoidance Task" (Rinck \& Becker, 2007) for our purposes by creating two conditions. In the "push negative away" condition, when participants saw a negative image, they were asked to push the negative image away. The image then shrank physically on the screen until it vanished. When a neutral image was shown to the participants, they were asked to pull the image toward themselves. The image then grew physically on the screen until it vanished. In the "pull negative closer" condition, when participants saw a negative image, they were asked to pull the negative image toward themselves. The image then grew physically on the screen as it was pulled closer until it vanished. When a neutral image was shown to the participants, they were asked to push the image away. The image then shrank physically on the screen until it vanished. We included neutral images so that participants performed the same movements, regardless of condition.

The image set for the actual experiment included 33 negative images and 33 neutral images selected from the International Affective Picture Set (IAPS; Lang, Bradley, \& Cuthbert, 2008). The images were matched for content, with each set consisting of 18 photos of people and 15 of objects. Images from IAPS 2007 that were rated as most negative were selected, excluding those with overly disturbing content (e.g., mutilation) and culturally specific 
content (e.g., Ku Klux Klan rallies). Images that were in the middle range of the valence scale (e.g., chair) were selected as the neutral images.

Momentary Avoided and Actual Affect: To assess whether the manipulation altered avoided negative affect, participants were asked how much they wanted to avoid feeling and how much they actually felt different states at that moment. We used the same affect words that we used for the AVI in Studies 2 and 3. Participants made their ratings on a 5-point scale ranging from 1 ("not at all") to 5 ("extremely"). Participants rated avoided affect before actual affect. To maintain consistency with Studies 2-3, responses were mean deviated within each individual by subtracting each individual's overall mean response to all 37 of the actual (or all 37 of the avoided) affect items from the raw score for each actual (or avoided) item. As in Study 3, we then computed mean aggregate scores for the negative items for actual and avoided affect (sad, unhappy, lonely, fearful, hostile, nervous, dull, sleepy, and sluggish). Internal consistencies (Cronbach's alphas) for avoided and actual negative affect were .84 and .78 for Americans and .96 and .59 for Germans, respectively.

Procedure-In the US, participants were recruited via a website offering students research credit for a large introductory psychology class at a large top-tier university. In Germany, participants were recruited from a large top-tier university via email announcements and flyers offering them a Euro 7 Amazon gift certificate. The study was advertised as a "reaction time and consumer products study."

American participants received all instructions and measures in English. German participants received all instructions and measures in German. The same translation-back translation technique was used as described for Studies 2 and 3 to ensure equivalence of the procedure and measures.

When participants arrived at the lab, they were greeted by the experimenter (an American female student for American participants and a German female student for German participants). Then they were seated in front of a computer that had a joystick attached to it, and asked to complete the consent form as well as to use a 5-point rating scale ranging from 1 ("not at all") to 5 ("very") to answer two questions about their current mood: (1) "how negatively do you currently feel?" and (2) "how positively do you currently feel?"

Participants were then introduced to the task and asked to read the instructions on the computer screen. In the "push negative away" condition, participants were told, "For NEGATIVE images, PUSH the joystick AWAY from yourself. For NEUTRAL images, PULL the joystick TOWARD yourself." In the "pull negative closer" condition, participants were told, "For NEGATIVE images, PULL the joystick TOWARD yourself. For NEUTRAL images, PUSH the joystick AWAY from yourself." After 20 practice trials with different IAPS images, the participant started with the actual experiment, which was comprised of 132 trials.

Each picture was presented to the participant twice in a standard random order ([33 negative +33 neutral images] $* 2=132$ trials). However, no more than three images of the same valence appeared successively. At the beginning of each trial, participants saw an image of 
medium size on the computer screen. Then, depending on whether they pushed or pulled the joystick, this image shrank or grew in size. Following the task, participants completed selfreport measures of actual and avoided affect. At the end of the study, participants were asked what they thought was the purpose of the study. Many participants thought that the images were intended to elicit emotion, but nobody guessed that the joystick movements were supposed to help increase or decrease the desire to avoid negative affect.

\section{Data Analyses and Results}

To examine whether we were successful in our random assignment, we examined the emotional state of our participants at the beginning of the study. We conducted a 2 (Group: Americans, Germans) $\times 2$ (Condition: push negative away, pull negative closer) $\times 2$ (Valence: negative, positive) repeated measures ANOVA with Group and Condition as between-subjects factors and Valence as within-subjects factor. The only significant effect that emerged was for Valence. Across cultural groups and conditions, participants felt more positively $($ mean $=3.69, \mathrm{SE}=.10)$ than negatively (mean $=1.94, \mathrm{SE}=.10)$ at the beginning of the study, $F[1,56]=104.48, p<.001$, partial eta squared $=.65$. Because we did not find any differences between conditions, we assume that we were successful in our random assignment.

To test our hypotheses, we conducted a $2 \times 2$ (Group: Americans, Germans $\times$ Condition: Push negative away, Pull negative closer) ANCOVA for avoided negative affect, controlling for actual negative affect. There was a significant main effect of Condition $(F[1,57]=5.57$, $p=.02$, partial eta squared $=.09)$ as well as a significant main effect of $\operatorname{Group}(F[1,57]=$ $15.23, p<.001$, partial eta squared $=.21)$. The Condition $\times$ Group interaction was not significant $(F[1,57]=1.10, p=.30$, partial eta squared $=.02)$. As predicted, participants in the "push negative away" condition wanted to avoid feeling negative states more than participants in the "pull negative closer" condition (Push negative away: Mean $=1.05, \mathrm{SE}$ $=.10$; Pull negative closer: Mean $=.71, \mathrm{SE}=.11)$. Furthermore, Americans wanted to avoid feeling negative states more than Germans did (Americans: $\mathrm{Mean}=1.28, \mathrm{SE}=.12$;

Germans: Mean $=.47, \mathrm{SE}=.13$ ).

We also examined whether participants in the two conditions and groups differed in their actual negative affect. We conducted a $2 \times 2$ (Group: Americans, Germans $\times$ Condition: Pull negative closer, Push negative away) ANCOVA for actual negative affect, controlling for avoided negative affect. Participants in the two conditions did not significantly differ in how much they actually felt negative (Push negative away: Mean $=-.49, \mathrm{SE}=.08$; Pull negative closer: Mean $=-.56, \mathrm{SE}=.09 ; F[1,57]=.30, p=.59$, partial eta squared $=.01)$. Thus, we were successful in selectively manipulating avoided negative affect (and not manipulating actual negative affect) with this task. However, we did find a significant main effect of Group: across conditions, Americans actually felt more negative than Germans did (Americans: Mean = -.03, SE = .09; Germans: Mean $=-1.02, \mathrm{SE}=.09 ; F[1,57]=56.04, p$ $<.001$, partial eta squared $=.50$ ). Because there were no group differences in mood prior to the experiment, we infer that Americans experienced more negative affect during the task than did Germans. 
Next we examined whether manipulating avoided negative affect would alter how much participants preferred negative vs. positive sympathy cards.

\section{Study 4b: Effects of Manipulating Avoided Negative Affect on Expressions of Sympathy Hypotheses}

We conducted Study $4 \mathrm{~b}$ to examine whether there was a causal relationship between the desire to avoid negative affect and expressions of sympathy across cultures. Using the experimental manipulation that we tested in Study 4a, we predicted that, across cultural groups, participants in the "push negative away" condition would be less likely to choose a negative vs. positive sympathy card than participants in the "pull negative closer" condition. We also predicted that, across conditions, Americans would be less likely to choose a negative over a positive sympathy card compared to Germans.

\section{Method}

Participants-Fifty-four American students (49.09\% European American; 53.85\% female; mean age $=19.63, \mathrm{SE}=.34)$ and 48 German students $(58.33 \%$ female; mean age $=23.30, \mathrm{SE}$ $=.35$ ) participated in this study. Participants were recruited and compensated in the same way as in Study 4a and randomly assigned to either the "push negative away" condition (US: $\mathrm{n}=28$; Germany: $\mathrm{n}=24$ ) or the "pull negative closer" condition (US: $\mathrm{n}=26$; Germany: $\mathrm{n}=24)$. The two cultural groups did not differ in gender $\left(\chi^{2}[1,100]=.20, p=\right.$. 65 , Cramer's V = .05), but Germans were significantly older than Americans, $F[1,96]=$ $56.72, p<.001$, partial eta squared $=.37$. Therefore, we controlled for age in the analyses below when it emerged as significant covariate.

Procedure and Instruments-American participants received all instructions and measures in English. German participants received all instructions and measures in German. The same translation-back translation procedures described above were used to ensure equivalence of the procedure and measures.

When participants arrived at the lab, they were greeted by the experimenter (an American female student for American participants and a German female student for German participants) and followed the same procedure as described in Study 4a. However, right after participants completed the joystick reaction time task, instead of completing the measure of actual and avoided affect as in Study 4a, participants were asked to complete the second part of the study, in which they had to imagine that the father of one of their acquaintances had just died, and that they were going to send a card to this acquaintance. Participants were then presented with a pair of sympathy cards (containing one negative and one positive card) on the computer screen. The pair was the same for both Americans and Germans. The cover of the negative card was beige, had leaves and other foliage, and contained the phrases, "Thinking of you at this difficult time;" the inside of the negative card contained the phrases, "No one truly comprehends the loss that you feel, the sadness that you must be going through. With sympathy and understanding." The cover of the positive card was white, had leaves and other foliage, and contained the phrases, "As you remember your loved one. May the memory of the smile warm you like the sun. May the memory of the 
laughter echo in your heart;" the inside of the positive card contained the phrases, "May the light of your best memories enrich all of your days. With caring thoughts and sympathy." Participants were asked to choose the card they would send to their acquaintance (cards available upon request).

\section{Study 4b Data Analyses and Results}

To examine whether we were successful in our random assignment, we examined the emotional state of our participants at the beginning of the study. We conducted a 2 (Group: Americans, Germans) $\times 2$ (Condition: push negative away, pull negative closer) $\times 2$ (Valence: negative feelings, positive feelings) repeated measures ANOVA with Group and Condition as between-subjects factors and valence as within-subjects factor. As in Study 4a, the only significant effect that emerged was for Valence. Across cultural groups and conditions, participants felt more positively (mean $=3.82, \mathrm{SE}=.08$ ) than negatively (mean $=1.79, \mathrm{SE}=.08)$ at the beginning of the study, $F[1,96]=221.03, p<.001$, partial eta squared $=.70$. Because we did not find any differences between conditions, we assume that we were successful in our random assignment.

To test our hypotheses, we conducted a logistic regression analysis in which card choice (1 $=$ negative, $2=$ positive) was regressed onto Condition, Group, and the interaction of Group and Condition. Because the interaction was not significant $(p=.70)$, we removed it from the final analysis. We found significant main effects of Condition and Group. As predicted, participants in the "push negative away" condition were less likely to choose the negative (vs. positive) card than those in the "pull negative closer" condition, $B=1.28, S E=.50, p=$. 01. Furthermore, Americans were less likely to choose the negative (vs. positive) card than were Germans, $B=2.43, S E=.50, p<.001$, Nagelkerke R square for model $=.39$. The percentages of participants who chose the negative cards are shown in Figure $7 .{ }^{17}$

\section{Study 4 Summary}

In summary, when we experimentally altered the degree to which participants wanted to avoid negative affect, we also altered their likelihood of choosing a negative (vs. positive) sympathy card. Interestingly, Americans reported feeling more negative than Germans after viewing neutral and negative images during the joystick task (before the manipulation, the current mood did not differ between groups). It is possible that for Americans, watching negative images might be more distressing than for Germans, ironically because they want to avoid the negative more.

Because we observed cultural group differences in actual as well as avoided negative affect, it is possible that the group differences in choice of negative (vs. positive) sympathy card were due to cultural differences in actual negative affect. However, in Study 3, we found that the more people actually felt negative, the less comfortable they were sending a positive sympathy card. In this study we found that Americans actually felt more negative, but were more likely to choose the positive sympathy card. Therefore, it seems unlikely that the

\footnotetext{
${ }^{17}$ We also included another pair of more indigenous sympathy cards; that is, Americans chose between a positive and negative American card, whereas Germans chose between a positive and negative German card. The results were the same.
} 
cultural difference in choice of negative (vs. positive) cards was due to actual negative affect rather than avoided negative affect. Regardless, across cultures, our findings support our prediction that the more people want to avoid negative affect, the less likely they are to focus on the negative (vs. positive) when expressing sympathy to another person.

\section{General Discussion}

While many scholars have examined sympathy, compassion, and other feelings of concern about the suffering of another person, relatively few scholars have examined how expressions of sympathy vary across cultures. In four studies, we demonstrate that cultures differ in how much they focus on the negative vs. positive when expressing sympathy to an acquaintance who has lost a loved one, and that these differences are due in part to the degree to which cultures encourage their members to avoid negative affect. More specifically, Americans focused less on the negative and more on the positive compared to their German counterparts when responding to the death of an acquaintance's family member in a hypothetical scenario, in part because they in general wanted to avoid negative affect more than Germans did. This is one of the first studies to illustrate cultural differences in views of negative emotion and the effects of these differences on expressions of sympathy.

In Study 1, we compared the emotional content of American and German sympathy cards and observed that American sympathy cards contained fewer negative emotion words (but more positive emotion words), lesser encouragement/acknowledgement of grief (but more encouragement/wish for something positive), and fewer dying images (but more living images) than did German sympathy cards. Interestingly, American cards included similar amounts of positive and negative words, whereas German cards included significantly more negative than positive words. In part, this is because in the few cases when American cards acknowledged the suffering of the recipient of the card, they also encouraged the person to focus on the positive, presumably as a way of coping with their pain. German cards, in contrast, simply acknowledged the suffering and grief of the recipient of the card,

presumably because they view focusing on the negative as a way of coping with pain, and/or because they believe that no positive words of encouragement can reduce the pain. German and American baby cards did not differ in positive or negative content, raising the possibility that the observed cultural differences may be specific to primarily negative events, although future research is needed to test this hypothesis.

Sympathy cards---like other cultural products---both reflect and reinforce cultural ideas and practices. Because cards are consumer products (i.e., created by designers whose goal is to sell cards), we assume that their emotional content reflects what the designers and their consumers view as an appropriate response to someone who has lost a loved one. Thus, we assumed that Americans prefer to purchase and send sympathy cards that are less negative and more positive than their German counterparts. Indeed, in Study 3, Americans reported feeling less comfortable sending a negative card and more comfortable sending a positive card than did Germans, and a significantly smaller percentage of Americans than Germans chose at least one negative (vs. positive) card from each of the three pairs they were presented with. Indeed, in Study $4 \mathrm{~b}$, when given a choice between a more negative and a 
more positive sympathy card, Germans were more than three times more likely to choose the negative (vs. positive) card than Americans. Together, these findings suggest that cultures vary in what they consider an appropriate way of expressing sympathy. Whereas American culture focuses less on the negative and more on the positive, German culture focuses more on the negative and less on the positive.

The Role of Avoided Negative Affect in Expressions of Sympathy-Why do these differences exist? We argued and found support for the hypothesis that cultural differences in expressions of sympathy in part reflect cultural differences in the degree to which people want to avoid feeling negative. Specifically, we demonstrated in Studies 3 and 4 that the degree to which people's expressions of sympathy focus on the negative (vs. positive) depends in part on how much they want to avoid negative emotion: the more people want to avoid negative emotion, the less they focus on the negative when responding to another person's pain. Americans wanted to avoid negative emotion more than Germans did, and these differences partly explained why Americans focused on the negative less when expressing concern for another. Moreover, when we experimentally altered avoided negative affect, we observed differences in preferences for negative (vs. positive) sympathy card across cultures. Participants who were experimentally induced to want to avoid negative states more were less likely to choose the negative (vs. positive) card. Although previous work has demonstrated that views of negative emotion impact how people respond to different stimuli (e.g., whether they want to see fear photographs after watching a fear film; Harmon-Jones et al. (2011) and other outcomes (e.g., Andrade \& Cohen, 2007; Grossmann \& Kross, 2010; Williams et al., 1997)), this is the first work to demonstrate how views of negative emotion influence expressions of sympathy.

Cultural Differences in Avoided Negative Affect-This work is also the first to examine the possible sources of cultural differences in the desire to avoid negative states. We predicted that because American culture more strongly endorses "frontier" values of achieving one's goals, influencing others, and overcoming nature than German culture (Koopmann-Holm \& Matsumoto, 2011; Schwartz \& Ros, 1995), American culture wants to avoid negative affect more than German culture. The founding fathers of the United States fled Europe to escape religious persecution, famine, and economic hardship (e.g., Lipset, 1997; Tucker, 1980; Wilson, 2008): in essence, they responded to their negative life circumstances by dreaming of a brighter future in an unknown wilderness and by pursuing that dream. They escaped the negative and focused on a positive future (Wilson, 2008). In contrast, their European counterparts remained in Europe, and to some degree accepted their life circumstances. In line with other scholars (e.g., Kitayama et al., 2006; Kitayama et al., 2009; Lipset, 1997; Tucker, 1980; Turner, 1921), we predicted that the psychological differences between the early settlers in the United States and their counterparts who stayed in Europe would be evident in the values endorsed by today's European Americans and Germans. Indeed, we observed that Americans endorsed specific frontier values more than their German counterparts did, and that these differences partly mediated cultural differences in avoided negative affect. However, this is only one possible explanation for the American-German differences in avoided negative affect, and future research is needed to examine other possible explanations. 
As mentioned above, this is one of the few studies comparing emotional processes in two individualistic Western cultures. These comparisons are critical because most of the studies on culture and emotion have focused on European American and East Asian comparisons. As argued by several scholars (Koopmann-Holm \& Matsumoto, 2011; Schwartz \& Ros, 1995), individualism/independence and collectivism/interdependence come in different forms, and different cultural value dimensions may be needed to flesh out these different forms. For instance, the value placed on overcoming vs. fitting into nature may be more relevant in American-European than in European American-East Asian comparisons.

Implications for Affect Valuation Theory-The present work expands AVT (Tsai, 2007) to negative states. Based on AVT, we predicted that avoided negative affect would be distinct from actual negative affect; that cultural factors would shape avoided negative affect more than actual negative affect; and that avoided negative affect would alter expressions of sympathy above and beyond actual negative affect. We found some support for all three predictions. In Study 2, structural equation modeling demonstrated that in both American and German samples, avoided, ideal, and actual negative affect were distinct constructs, and that these differences held regardless of the type of negative affect (high, moderate, or low arousal) assessed.

AVT predicts that cultural differences in avoided negative affect would be greater than cultural differences in actual negative affect. We found support for this prediction as well. Whereas consistent cultural differences emerged in avoided negative affect across Studies 2-4, with Americans wanting to avoid negative affect more than Germans, cultural differences in actual negative affect were less consistent. In Study 2, European Americans and Germans did not differ in their experience of HAN (e.g., nervousness) or LAN (e.g., dullness), but European Americans experienced less N (e.g., sadness) than did Germans. In Study 3, Americans reported experiencing less negative affect overall than did Germans. In Study $4 \mathrm{a}$, although there were no cultural differences in actual negative affect at baseline, in response to the negative and neutral images, Americans reported experiencing more negative affect than Germans did.

Although it is unclear why differences in actual negative affect emerged in some cases and not others, it seems unlikely that these inconsistencies were due to stable cultural values. Indeed, in Study 3, frontier values mediated cultural differences in avoided negative affect but not in actual negative affect. In Tsai (2007), we suggested that although actual affect may be shaped by cultural factors, it is also influenced by people's temperaments, immediate circumstances, as well as their regulatory abilities. In Study 3, we suggested that the difference in actual negative affect might have been due to the different economic conditions in the US and Germany at the time of the study. In Study $4 \mathrm{a}$, it is possible that because Americans wanted to avoid negative states more than did Germans, they actually experienced the negative images more negatively than Germans did. Future research is needed to test these possible explanations.

In future studies, we hope to examine how ideal positive affect and avoided negative affect interact, and examine the circumstances under which one is more or less important in predicting behavior. For instance, it is possible that avoided negative affect drives behavior 
more in situations in which people already feel negative affective states or are anticipating feeling negative, whereas ideal positive affect may drive behavior more when people are in a neutral state.

Implications for Models of Sympathy, Compassion, and Helping-Existing models of helping assume that people help (or not) because they want to reduce their experiences of negative affect (Batson, 1981, 1983; Batson et al., 1991; Cialdini et al., 1973; Cialdini et al., 1987). Our findings suggest that cultures and individuals vary in their desire to avoid negative affect, and that these differences shape how they respond to the suffering of another person. Thus, our research suggests that models of helping should not just describe whether or not people help, but also how they help. While current models of helping may hold in contexts in which individuals want to avoid negative affect more, they may be less applicable to cultural contexts in which individuals want to avoid negative states less. In other words, wanting to reduce negative affect may motivate helping more in American than in German contexts. Future work is needed to test these ideas, with the goal of developing models of helping, sympathy, and compassion that are sensitive to different cultural ideals and norms regarding emotion.

Implications for Clinical Assessment and Treatment-Our findings also have important implications for assessing and treating emotional distress across cultures. For instance, it is possible that depression (and other disorders that are characterized by negative affect) is more often diagnosed in cultures that want to avoid feeling negative affect more. Indeed, depression is more often diagnosed in the US compared to Germany (Bromet et al., 2011). Similarly, cultural differences in the desire to avoid negative affect may result in different criteria for diagnoses of depression and other affective disorders. For instance, with the release of the $5^{\text {th }}$ edition of the American Psychiatric Association's Diagnostic and Statistical Manual of Mental Disorders (DSM-V), debate emerged about whether the DSM$\mathrm{V}$ should continue to exclude depressive symptoms due to bereavement when diagnosing a major depressive episode. While many American clinicians wanted to allow bereaved individuals to receive a diagnosis of major depression, numerous European clinicians and scientists felt depressive symptoms due to bereavement should not be considered in diagnoses of depression. As one German newspaper stated, "Trauernde [...] müssen davor bewahrt werden, als krank zu gelten", or "people who are grieving [...] must not be labeled as ill" (Simon, 2011).

Cultural differences in avoided negative affect also have implications for the treatment of disorders such as depression. Clinicians may develop treatments that reflect their own culture's view of negative affect. For instance, Sigmund Freud, the father of psychoanalysis, was Austrian and thus influenced by German culture. Given the greater acceptance of negative emotion in German contexts, it is not surprising that a core facet of psychoanalysis is the notion that suppressing and avoiding negative emotions is bad and that talking about one's negative emotions is therapeutic (Freud, 1977). Similarly, given the greater avoidance of negative emotion in American contexts, it is perhaps not surprising that American psychiatrist Aaron Beck developed cognitive therapy (Beck, 1979) as a reaction to psychoanalysis, and emphasized teaching patients various "mood repair strategies" (e.g., 
retrieving positive memories when one is sad) so that they could shift their mood from negative to positive. Indeed, this may explain why repressive coping, which was viewed as dysfunctional by Freud, actually seems functional in American contexts (Coifman, Bonanno, Ray, \& Gross, 2007). Similarly, this may explain why antidepressants are used more often in the US than in Germany (e.g., Zito et al., 2008).

These findings may be particularly important for grief and trauma counseling, which, in our coalescing world, is often organized and provided internationally, without understanding the norms of the culture in need (Watters, 2010). Counselors providing assistance in other cultures must know whether to encourage their patients to tell their "sad heart [to] cease repining [because] behind the clouds ... the sun [is] still shining" or to "pass the night in tears, as long as [they] want to cry."

Limitations and Current Directions-These studies have a number of limitations that pave the way for future research. First, it is unclear whether cultural differences in avoided negative affect mediate expressions of sympathy that focus on the negative and the positive, or just the negative. Although Study 3 suggests that it may be just the negative, in Study $4 \mathrm{~b}$, we cannot distinguish the two because participants were given a choice between a negative and positive card. In conducting these studies, we considered adding a "neutral" sympathy card; however, pilot testing revealed how difficult this was. For instance, cards that appeared neutral in American contexts (e.g., a card with green leaves) were perceived as positive in German contexts. Nevertheless, this would be an important direction to pursue in future research.

Second, we focused on how cultural and individual differences in avoided negative affect influence how people hypothetically respond to the suffering of an acquaintance; in future studies, it would be important to examine whether these findings generalize to other relationships (e.g., the suffering of a family member or friend), to actual (vs. hypothetical) negative events, and to how people respond to their own grief as well as how they would like others to respond to their own grief (e.g., which kind of sympathy cards they would like to receive from others). For instance, Clark (1997), in her analysis of American sympathy, observes that recipients of sympathy are expected to count their "blessings," or focus on the positive. We predict that there would be less of this expectation in German contexts. We have already started to examine these questions in our current research. In the future, it would be also important to examine how avoided negative affect and actual negative affect vary across situations and across time (i.e., how people feel immediately after learning about another person's distress vs. weeks later). Similarly, it will be important to examine whether cultural differences in avoided negative affect alter expressions of other emotions such as anger and influence responses to other types of negative events (e.g., illness, difficulties at a job).

Finally, Study 3 only began to examine the causes of cultural differences in the desire to avoid negative affect by focusing on specific frontier values. Certainly, alternative explanations for American-German differences are possible and should be tested in the future. Furthermore, because the present work was correlational, future studies should use experimental methods to examine whether endorsing frontier values results in a greater 
desire to avoid negative affect, or whether the reverse is true. Future work should also examine whether these differences are unique to American vs. German comparisons--ethnographic evidence suggests that other European cultures may also be more accepting of negative emotions than mainstream North American culture (e.g., Gaines \& Farmer, 1986; Grossmann \& Kross, 2010). Such studies would also provide support for the notion that there are different forms of individualism and independence. Similarly, the present work focused on European Americans, but it would be interesting to examine whether other ethnic groups in the United States who have a similar voluntary settlement history also differ from their counterparts who chose not to immigrate to the United States in terms of their views of negative emotion and expressions of sympathy.

Conclusion-Does culture shape how people express their sympathy for another person? In a series of four studies, we demonstrate that American expressions of sympathy focus less on the negative and more on the positive than German expressions of sympathy, and that these differences are due in part to the degree to which Americans want to avoid negative affect. These studies demonstrate the importance of considering cultural views of emotion in current models of sympathy, compassion, and helping.

\section{Acknowledgments}

Birgit Koopmann-Holm and Jeanne L. Tsai, Department of Psychology, Stanford University. This work is based on the dissertation of the first author. This research was funded by the Stanford Graduate Research Opportunities and the Norman Anderson Research Fund to the first author and NIMH grant R01MH068879, NIA grant R03 AG023302, and NSF grant BCS-1324461 to the second author. We would like to thank Hazel Markus, James Gross, Brian Knutson, Jennifer Aaker, Ewart Thomas, the Stanford Culture and Emotion Laboratory, and the Stanford Culture Collaboratory for their invaluable feedback. We would also like to thank Meike Uhrig and Pamela Scholz for coding the cards from Study 1 and Julia Görnandt, Kathrin Gählert as well as Sabine Krueger for their help with the translations. We also want to thank Kathrin Gählert, Jenny Jordan, Zoe Wolford, Anny Dow, Parth Patel, Chrissy Scannell, and Isabelle Hofsäß for running participants and Fanja Riedel, Annette Kämmerer, and Peter Fiedler for their support in the data collection in Germany; Mike Rinck for sending us the "ApproachAvoidance Task" program; the German greeting card companies bsb, Hallmark, Hanra, and Taurus for generously sending us the cards and card catalogues, and to Linda Calvillo for compiling the American Hallmark cards. Finally, B.K.-H. thanks Brian Holm, Milena Holm, Damian Holm, Gerlinde Koopmann, Dietrich Koopmann, Jan Koopmann, Barbara Capron, Bruce Capron, and Saskia Lang for their support during the preparation of this article.

\section{References}

Anderson ML. Embodied cognition: A field guide. Artificial intelligence. 2003; 149(1):91-130.

Andrade EB, Cohen JB. On the consumption of negative feelings. Journal of Consumer Research. 2007; 34(3):283-300.

Baron RM, Kenny DA. The moderator-mediator variable distinction in social psychological research: Conceptual, strategic, and statistical considerations. Journal of personality and social psychology. 1986; 51(6):1173-1182. [PubMed: 3806354]

Batson CD. Is empathic emotion a source of altruistic motivation? Journal of personality and social psychology. 1981; 40(2):290-302.

Batson CD. Influence of self-reported distress and empathy on egoistic versus altruistic motivation to help. Journal of personality and social psychology. 1983; 45(3):706-718.

Batson CD, Batson J, Griffitt C, Barrientos S, Brandt J, Sprengelmeyer P, Bayly M. Negative-state relief and the empathy-altruism hypothesis. Journal of Personality and Social Psychology. 1989; 56:922-933.

Batson CD, Batson JG, Slingsby JK, Harrell KL, Peekna HM, Todd RM. Empathic joy and the empathy-altruism hypothesis. Journal of Personality and Social Psychology. 1991; 61:413-426. [PubMed: 1941512] 
Beck, AT. Cognitive therapy of depression. The Guilford Press; 1979.

Brainerd C, Stein L, Silveira R, Rohenkohl G, Reyna V. How does negative emotion cause false memories? Psychological Science. 2008; 19(9):919-925. [PubMed: 18947358]

Bromet E, Andrade LH, Hwang I, Sampson NA, Alonso J, de Girolamo G, Iwata N. Cross-national epidemiology of DSM-IV major depressive episode. BMC medicine. 2011; 9(1):90. [PubMed: 21791035]

Brooks, D. On Paradise Drive: How We Live Now (and Always Have) in the Future Tense. Simon \& Schuster; New York: 2004.

Canfield, J.; Hansen, MV.; Tabatsky, D. Chicken Soup for the Soul: The Cancer Book: 101 Stories of Courage, Support, and Love. Chicken Soup for the Soul Publishing; New York: 2009.

Chambless DL, Caputo GC, Bright P, Gallagher R. Assessment of fear of fear in agoraphobics: The Body Sensations Questionnaire and the Agoraphobic Cognitions Questionnaire. Journal of Consulting and Clinical Psychology. 1984; 52(6):1090-1097. [PubMed: 6520279]

Cheung GW, Rensvold RB. Testing factorial invariance across groups: A reconceptualization and proposed new method. Journal of Management. 1999; 25(1):1-27.

Chorpita BF, Albano AM, Barlow DH. The structure of negative emotions in a clinical sample of children and adolescents. Journal of Abnormal Psychology. 1998; 107(1):74-85. [PubMed: 9505040]

Cialdini RB, Darby BL, Vincent JE. Transgression and altruism: A case for hedonism. Journal of Experimental Social Psychology. 1973; 9:502-516.

Cialdini RB, Schaller M, Houlihan D, Arps K, Fultz J, Beaman A. Empathy-based helping: Is it selflessly or selfishly motivated. Journal of Personality and Social Psychology. 1987; 52:749-758. [PubMed: 3572736]

Clair, J. Melancholie: Genie und Wahnsinn in der Kunst [Melancholy: Genius and madness in the arts]. Hatje Cantz; Ostfilden-Ruit: 2005.

Clark, C. Misery and company: Sympathy in everyday life. University of Chicago Press; Chicago, IL: 1997.

Coifman KG, Bonanno GA, Ray RD, Gross JJ. Does repressive coping promote resilience? Affectiveautonomic response discrepancy during bereavement. Journal of personality and social psychology. 2007; 92(4):745-758. [PubMed: 17469956]

Condon P, DeSteno D. Compassion for one reduces punishment for another. Journal of Experimental Social Psychology. 2011; 47(3):698-701.

Condon P, Feldman Barrett L. Conceptualizing and Experiencing Compassion. 2013

Conway, LG., III; Houck, SC.; Gornick, LJ. Regional differences in individualism and why they matter.. In: Rentfrow, PJ., editor. Geographical Psychology: Exploring the Interaction of Environment and Behavior. American Psychological Association; Washington, DC: 2014. p. 31-50.

Costa PT, McCrae RR. Influence of extraversion and neuroticism on subjective well-being: Happy and unhappy people. Journal of Personality and Social Psychology. 1980; 38(4):668-678. [PubMed: 7381680]

Dai X, Brendl CM, Ariely D. Wanting, liking, and preference construction. Emotion. 2010; 10(3):324334. [PubMed: 20515222]

David JP, Green PJ, Martin R, Suls J. Differential roles of neuroticism, extraversion, and event desirability for mood in daily life: An integrative model of top-down and bottom-up influences. Journal of Personality and Social Psychology. 1997; 73(1):149-159. [PubMed: 9216082]

Davis M. Measuring individual differences in empathy: Evidence for a multidimensional approach. Journal of Personality and Social Psychology. 1983; 44:113-126.

de Greck M, Shi Z, Wang G, Zuo X, Yang X, Wang X, Han S. Culture modulates brain activity during empathy with anger. Neuroimage. 2012; 59(3):2871-2882. [PubMed: 21983057]

Diener, E.; Lucas, R. Personality and subjective well-being.. In: Kahneman, D.; Diener, E.; Schwarz, N., editors. Well-being: Foundations of hedonic psychology. Russell Sage Foundation; New York: 1999. p. 213-229. 
Eddy, M.; Jolly, D. Unemployment rises to new high in Euro zone. The New York Times; Jan 8. 2013 Retrieved from http://www.nytimes.com

Ehrenreich, B. Bright-sided: How the relentless promotion of positive thinking has undermined America. Metropolitan Books; New York: 2009.

Eid M, Diener E. Norms for experiencing emotions in different cultures: Inter-and international differences. Journal of personality and social psychology. 2001; 81(5):869-885. [PubMed: 11708563]

Eisenberg N, Fabes RA, Miller PA, Fultz J, Shell R, Mathy RM, Reno RR. Relation of sympathy and personal distress to prosocial behavior: a multimethod study. Journal of personality and social psychology. 1989; 57(1):55-66. [PubMed: 2754604]

Eisenberg N, Fabes RA, Murphy B, Karbon M, Maszk P, Smith M, Suh K. The relations of emotionality and regulation to dispositional and situational empathy-related responding. Journal of personality and social psychology. 1994; 66(4):776-797. [PubMed: 8189352]

Eisenberg N, Fabes RA, Shepard SA, Murphy BC, Jones S, Guthrie IK. Contemporaneous and longitudinal prediction of children's sympathy from dispositional regulation and emotionality. Developmental Psychology. 1998; 34(5):910-924. [PubMed: 9779738]

Eisenberg N, Miller P. The relation of empathy to prosocial and related behaviors. Psychological Bulletin. 1987; 101(1):91-119. [PubMed: 3562705]

Eisenberg N, Zhou Q, Koller S. Brazilian Adolescents' Prosocial Moral Judgment and Behavior: Relations to Sympathy, Perspective Taking, Gender-Role Orientation, and Demographic Characteristics. Child Development. 2001; 72(2):518-534. [PubMed: 11333082]

Forgas JP. Affect and cognition. Perspectives on Psychological Science. 2008; 3(2):94-101.

Frasure-Smith N, Lesperance F, Talajic M. The impact of negative emotions on prognosis following myocardial infarction: is it more than depression? Health Psychology. 1995; 14(5):388-398. [PubMed: 7498109]

Freud, S. Introductory lectures on psycho-analysis. Vol. 1. WW Norton \& Company; 1977.

Gaines AD, Farmer PE. Visible saints: Social cynosures and dysphoria in the Mediterranean tradition. Culture, Medicine and Psychiatry. 1986; 10(4):295-330.

Gelfert, H-D. Was ist deutsch? Wie die Deutschen wurden, was sie sind [What is German? How the Germans became the way they are]. C. H. Beck Verlag; Munich: 2005.

Goetz JL, Keltner D, Simon-Thomas E. Compassion: an evolutionary analysis and empirical review. Psychological Bulletin. 2010; 136(3):351-374. [PubMed: 20438142]

Gross JJ. The emerging field of emotion regulation: An integrative review. Review of General Psychology. 1998; 2:271-299.

Gross JJ, Carstensen LL, Pasupathi M, Tsai JL, Skorpen CG, Hsu AY. Emotion and aging: Experience, expression, and control. Psychology and Aging. 1997; 12:590-599. [PubMed: 9416628]

Gross JJ, Levenson RW. Hiding feelings: The acute effects of inhibiting negative and positive emotion. Journal of Abnormal Psychology. 1997; 106:95-103. [PubMed: 9103721]

Gross JJ, Sutton SK, Ketelaar T. Relations between affect and personality: Support for the affect-level and affective reactivity views. Personality and Social Psychology Bulletin. 1998; 24(3):279-288.

Grossmann I, Kross E. The impact of culture on adaptive versus maladaptive self-reflection. Psychological Science. 2010; 21(8):1150-1157. [PubMed: 20624935]

Han S-P, Shavitt S. Persuasion and culture: Advertising appeals in individualistic and collectivistic societies. Journal of Experimental Social Psychology. 1994; 30:326-326.

Harmon-Jones E, Harmon-Jones C, Amodio DM, Gable PA. Attitudes toward emotions. Journal of personality and social psychology. 2011; 101(6):1332-1350. [PubMed: 21843012]

Hayes SC, Strosahl K, Wilson KG, Bissett RT, Pistorello J, Toarmino D, Bergan J. Measuring experiential avoidance: A preliminary test of a working model. The psychological record. 2004; 54(4):553-578.

Hedderich N. When cultures clash: Views from the professions. Die Unterrichtspraxis/Teaching German. 1999:158-165. 
Held BS, Bohart AC. Introduction: The (overlooked) virtues of "unvirtuous" attitudes and behavior: Reconsidering negativity, complaining, pessimism, and "false" hope. Journal of Clinical Psychology. 2002; 58(9):961-964. [PubMed: 12209858]

Henneberger, M. Not all cancer survivors 'mesmerized' by Lance Armstrong, The Washington Post. Jan 15. 2013 Retrieved from http://www.washingtonpost.com

Hertenstein MJ, Keltner D, App B, Bulleit BA, Jaskolka AR. Touch communicates distinct emotions. Emotion. 2006; 6(3):528-533. [PubMed: 16938094]

Izard, CE. The face of emotion. Appleton-Century-Crofts; New York: 1971.

Jiang C, Varnum ME, Hou Y, Han S. Distinct effects of self-construal priming on empathic neural responses in Chinese and Westerners. Social neuroscience. 2014; 9(2):130-138. [PubMed: 24341541]

Keltner, D. Born to be good: The science of a meaningful life. WW Norton \& Company; 2009.

Kenny, DA.; Kashy, DA.; Bolger, N. Data analysis in social psychology.. In: Gilbert, DT.; Fiske, ST.; Lindzey, G., editors. The handbook of social psychology. 4th ed.. Vol. 1. Oxford University Press; New York: 1998. p. 233-265.

Kiefer T. Feeling bad: Antecedents and consequences of negative emotions in ongoing change. Journal of Organizational Behavior. 2005; 26(8):875-897.

Kitayama S, Ishii K, Imada T, Takemura K, Ramaswamy J. Voluntary Settlement and the Spirit of Independence: Evidence from Japan's 'Northern Frontier'. Journal of Personality and Social Psychology. 2006; 91:369-384. [PubMed: 16938025]

Kitayama, S.; Markus, HR. The pursuit of happiness and the realization of sympathy: Cultural patterns of self, social relations, and well-being.. In: Diener, E.; Suh, E., editors. Subjective well-being across cultures. MIT Press; Cambridge, MA: 2000. p. 113-161.

Kitayama S, Markus HR, Kurokawa M. Culture, emotion, and well-being: Good feelings in Japan and the United States. Cognition \& Emotion. 2000; 14:93-124.

Kitayama S, Park H, Sevincer AT, Karasawa M, Uskul AK. A cultural task analysis of implicit independence: comparing North America, Western Europe, and East Asia. Journal of personality and social psychology. 2009; 97(2):236-255. [PubMed: 19634973]

Koopmann-Holm B, Matsumoto D. Values and display rules for specific emotions. Journal of CrossCultural Psychology. 2011; 42(3):355-371.

Kuppens P, Realo A, Diener E. The role of positive and negative emotions in life satisfaction judgment across nations. Journal of personality and social psychology. 2008; 95(1):66-75. [PubMed: 18605852]

Lamoreaux M, Morling B. Outside the Head and Outside Individualism-Collectivism Further MetaAnalyses of Cultural Products. Journal of Cross-Cultural Psychology. 2012; 43(2):299-327.

Landis JR, Koch GG. The measurement of observer agreement for categorical data. Biometrics. 1977; 33(1):159-174. [PubMed: 843571]

Lang, PJ.; Bradley, M.; Cuthbert, BN. Technical Report A-8. University of Florida; Gainesville, FL.: 2008. International affective picture system (IAPS): Affective ratings of pictures and instruction manual..

Lennon, R.; Eisenberg, N. Gender and age differences in empathy and sympathy.. In: Eisenberg, N.; Strayer, J., editors. Empathy and its development. Cambridge University Press; New York: 1987. p. 195-217.

Lesch M. Ein Jahr Hölle: Bastei Lübbe. 2008

Levine R, Norenzayan A, Philbrick K. Cross-cultural differences in helping strangers. Journal of Cross-Cultural Psychology. 2001; 32(5):543-560.

Lipset, S. American exceptionalism: A double-edged sword. WW Norton \& Company; 1997.

Little TD. Mean and covariance structures (MACS) analyses of cross-cultural data: Practical and theoretical issues. Multivariate Behavioral Research. 1997; 32(1):53-76.

Lockwood P, Jordan CH, Kunda Z. Motivation by positive or negative role models: Regulatory focus determines who will best inspire us. Journal of Personality and Social Psychology. 2002; 83:854864. [PubMed: 12374440] 
Lykken D, Tellegen A. Happiness is a stochastic phenomenon. Psychological Science. 1996; 7:186189.

MacKinnon DP, Lockwood CM, Hoffman JM, West SG, Sheets V. A comparison of methods to test mediation and other intervening variable effects. Psychological Methods. 2002; 7:83-104. [PubMed: 11928892]

Maio GR, Esses VM. The need for affect: Individual differences in the motivation to approach or avoid emotions. Journal of Personality. 2001; 69:583-615. [PubMed: 11497031]

Markus HR, Uchida Y, Omoregie H, Townsend SS, Kitayama S. Going for the Gold Models of Agency in Japanese and American Contexts. Psychological Science. 2006; 17(2):103-112. [PubMed: 16466417]

McAdams D. The redemptive self: Narrative identity in America today. The self and memory. 2004:95-116.

McCrae, RR.; Costa, PT.; Yik, M. Universal aspects of Chinese personality structure.. In: Bond, MH., editor. The handbook of Chinese psychology. Oxford University Press; New York: 1996. p. 189-207.

Mesquita, B.; Markus, HR. Culture and emotion: Models of agency as sources of cultural variation in emotion.. In: Frijda, NH.; Manstead, ASR.; Fisher, A., editors. Feelings and emotions: The Amsterdam symposium. Cambridge University Press; Cambridge, MA: 2004. p. 341-358.

Miller JG, Bersoff DM. Cultural influences on the moral status of reciprocity and the discounting of endogenous motivation. Personality and Social Psychology Bulletin. 1994; 20(5):592-602.

Morling B, Lamoreaux M. Measuring culture outside the head: A meta-analysis of individualismcollectivism in cultural products. Personality and Social Psychology Review. 2008; 12(3):199_ 221. [PubMed: 18544712]

Nussbaum M. Compassion: The basic social emotion. Social Philosophy and Policy. 1996; 13:27-58.

Oveis C, Horberg E, Keltner D. Compassion, pride, and social intuitions of self-other similarity. Journal of personality and social psychology. 2010; 98(4):618-630. [PubMed: 20307133]

Pennebaker, JW.; Francis, ME.; Booth, RJ. Linguistic inquiry and word count: LIWC 2001. Lawrence Erlbaum Associates; Mahway: 2001.

Rinck M, Becker ES. Approach and avoidance in fear of spiders. Journal of behavior therapy and experimental psychiatry. 2007; 38(2):105-120. [PubMed: 17126289]

Rozin P. Five potential principles for understanding cultural differences in relation to individual differences. Journal of Research in Personality. 2003; 37:273-283.

Russell JA. A circumplex model of affect. Journal of Personality and Social Psychology. 1980; 39:1161-1178.

Russell JA, Lewicka M, Niit T. A cross-cultural study of a circumplex model of affect. Journal of Personality and Social Psychology. 1989; 57:848-856.

Rusting CL, Larsen RJ. Extraversion, neuroticism, and susceptibility to positive and negative affect: A test of two theoretical models. Personality and Individual Differences. 1997; 22(5):607-612.

Schimmack U, Radhakrishnan P, Oishi S, Dzokoto V, Ahadi S. Culture, personality, and subjective well-being: Integrating process models of life satisfaction. Journal of Personality and Social Psychology. 2002; 82(4):582-593. [PubMed: 11999925]

Schwartz, SH. Universals in the content and structure of values: Theoretical advances and empirical tests in 20 countries.. In: Zanna, MP., editor. Advances in experimental social psychology. Vol. 25. Academic Press; Orlando, FL: 1992. p. 1-65.

Schwartz SH. An overview of the Schwartz theory of basic values. Online Readings in Psychology and Culture. 2012; 2(1) doi: http://dx.doi.org/10.9707/2307-0919.1116.

Schwartz SH, Ros M. Values in the west: A theoretical and empirical challenge to the individualismcollectivism cultural dimension. World Psychology. 1995; 1:91-122.

Shaver PR, Murdaya U, Fraley RC. Structure of the Indonesian emotion lexicon. Asian journal of social psychology. 2001; 4(3):201-224.

Shweder, RA. Why do men barbecue? Recipes for cultural psychology. Harvard University Press; Cambridge, MA: 2003. 
Simon, N. Psychiatrie für alle, Taz.. Die Tageszeitung. May 13. 2011 Retrieved from http:// www.taz.de

Sommers, S. Adults evaluating their emotions: A cross-cultural perspective.. In: Malatesta, CZ.; Izard, CE., editors. Emotion in adult development. Sage; Beverly Hills, CA: 1984. p. 319-338.

Stearns, PN. American cool: Constructing a twentieth-century emotional style. New York University Press; 1994.

Stellar JE, Manzo VM, Kraus MW, Keltner D. Class and compassion: Socioeconomic factors predict responses to suffering. Emotion. 2012; 12(3):449-459. [PubMed: 22148992]

Tamir M, Ford BQ. Choosing to be afraid: Preferences for fear as a function of goal pursuit. Emotion. 2009; 9(4):488-497. [PubMed: 19653771]

Tamir M, Mitchell C, Gross JJ. Hedonic and instrumental motives in anger regulation. Psychological Science. 2008; 19(4):324-328. [PubMed: 18399883]

Taylor S, Rachman S. Fear of sadness. Journal of Anxiety Disorders. 1991; 5(4):375-381.

Toi M, Batson CD. More evidence that empathy is a source of altruistic motivation. Journal of Personality and Social Psychology. 1982; 43:281-292.

Trommsdorff G, Friedlmeier W, Mayer B. Sympathy, distress, and prosocial behavior of preschool children in four cultures. International Journal of Behavioral Development. 2007; 31(3):284-293.

Tsai JL. Ideal affect: Cultural causes and behavioral consequences. Perspectives on Psychological Science. 2007; 2:242-259.

Tsai JL, Knutson B. The Affect Valuation Index: Reliability and validity. 2006 Manuscript in preparation.

Tsai JL, Knutson B, Fung H. Cultural Variation in Affect Valuation. Journal of Personality and Social Psychology. 2006; 90:288-307. [PubMed: 16536652]

Tsai JL, Louie JY, Chen EE, Uchida Y. Learning what feelings to desire: Socialization of ideal affect through children's storybooks. Personality and Social Psychology Bulletin. 2007; 33(1):17-30. [PubMed: 17178927]

Tsai JL, Miao FF, Seppala E. Good feelings in Christianity and Buddhism: Religious differences in ideal affect. Personality and Social Psychology Bulletin. 2007; 33:409-421. [PubMed: 17312321]

Tsai JL, Miao FF, Seppala E, Fung H, Yeung D. Influence and adjustment goals: Sources of cultural differences in ideal affect. Journal of Personality and Social Psychology. 2007; 92:1102-1117. [PubMed: 17547491]

Tucker, FH. The frontier spirit and progress. Nelson-Hall; Chicago: 1980.

Turner, FJ. The frontier in American history. Holt; New York: 1921.

Valdesolo P, DeSteno D. Synchrony and the social tuning of compassion. Emotion. 2011; 11(2):262266. [PubMed: 21500895]

Van Kleef GA, Oveis C, Van der Löwe I, LuoKogan A, Goetz J, Keltner D. Power, Distress, and Compassion Turning a Blind Eye to the Suffering of Others. Psychological Science. 2008; 19(12):1315-1322. [PubMed: 19121143]

Varnum ME. Frontiers, germs, and nonconformist voting. Journal of Cross-Cultural Psychology. 2013; 44(5):832-837.

Varnum ME. Sources of regional variation in social capital in the United States: Frontiers and pathogens. Evolutionary Behavioral Sciences. 2014; 8(2):77-85.

Varnum ME, Kitayama S. What's in a name? Popular names are less common on frontiers. Psychological Science. 2011; 22(2):176-183. [PubMed: 21196534]

Västfjäll D, Gärling T. Preference for negative emotions. Emotion. 2006; 6:326-329. [PubMed: 16768564]

Watters, E. Crazy like us: The globalization of the American psyche. Simon and Schuster; New York: 2010.

Williams KE, Chambless DL, Ahrens A. Are emotions frightening? An extension of the fear of fear construct. Behaviour Research and Therapy. 1997; 35(3):239-248. [PubMed: 9125104]

Wilson, EG. Against happiness: In praise of melancholy. Sarah Crichton Books; New York: 2008. 
Wuthnow, R. Acts of compassion: Caring for others and helping ourselves. Princeton University Press; Princeton: 2012.

Zajonc RB. Mere exposure: A gateway to the subliminal. Current Directions in Psychological Science. 2001; 10(6):224-228.

Zaki J, Ochsner KN. The neuroscience of empathy: progress, pitfalls and promise. Nature neuroscience. 2012; 15(5):675-680.

Zhang A, Xia F, Li C. The antecedents of help giving in Chinese culture: Attribution, judgment of responsibility, expectation change and the reaction of affect. Social Behavior and Personality. 2007; 35(1):135-142.

Zito JM, Safer DJ, de Jong-van den Berg LT, Janhsen K, Fegert JM, Gardner JF, Valluri SC. A threecountry comparison of psychotropic medication prevalence in youth. Child and Adolescent Psychiatry and Mental Health. 2008; 2:26. [PubMed: 18817536] 


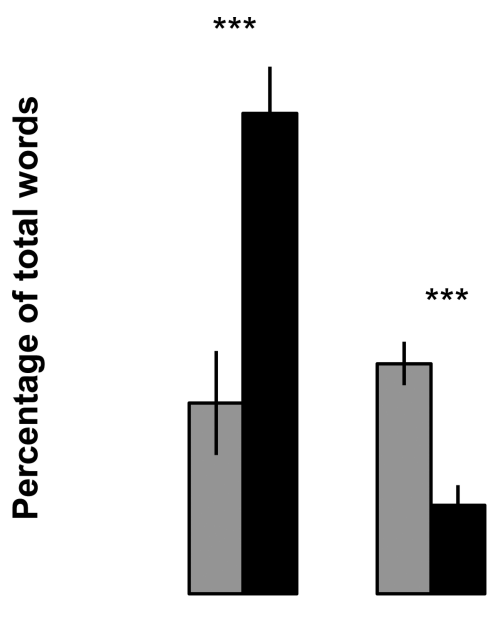

Sympathy Cards

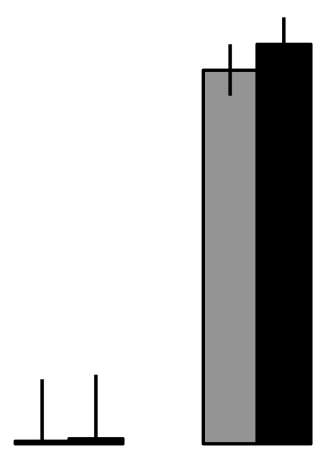

Baby Cards

1.

Frequency of negative words and positive words (percentage of total words that are negative or positive) in American and German sympathy and baby cards in Study 1 . 


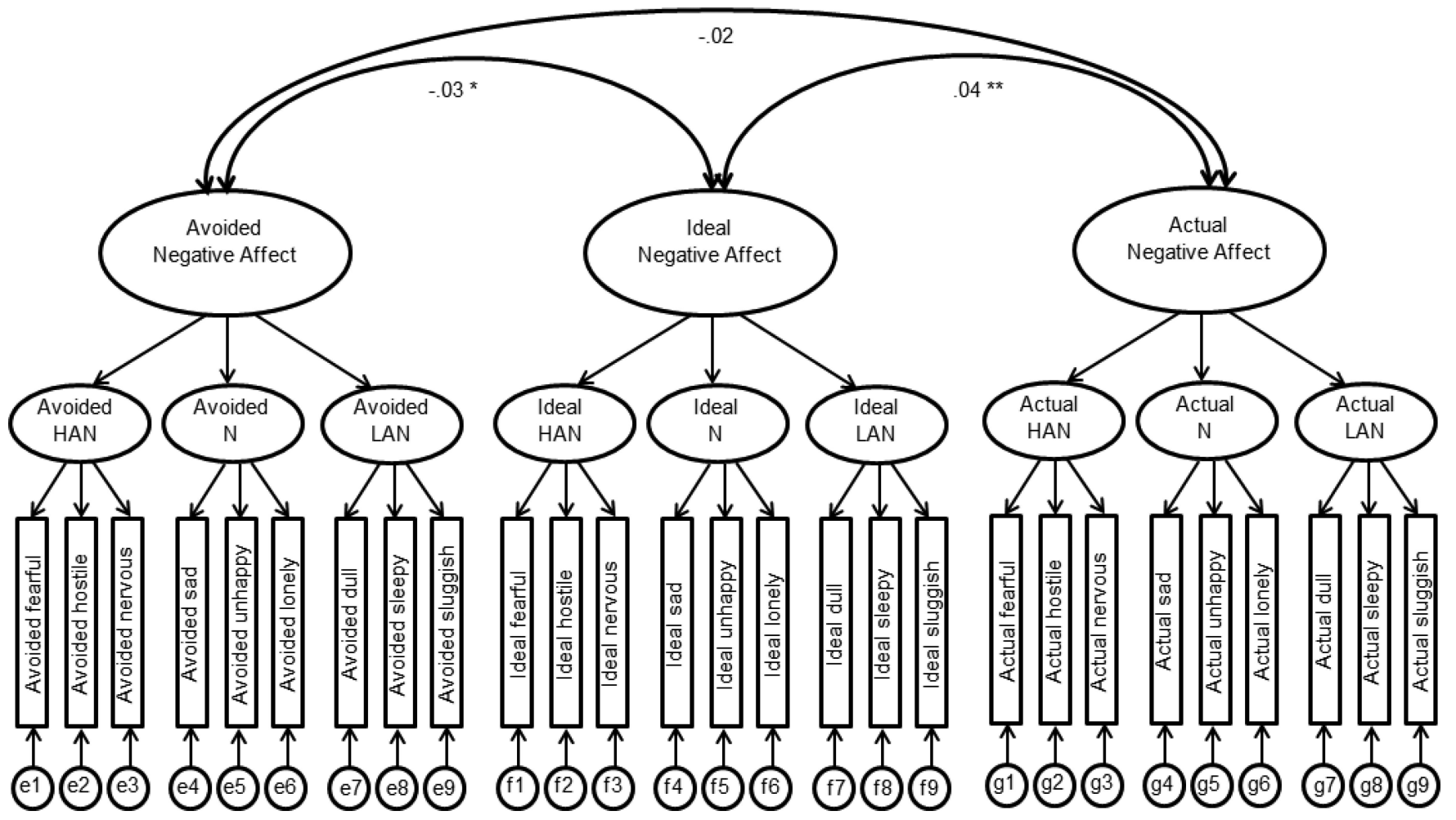

2.

Factor structure of ideal, actual, and avoided negative affect in Study 2. Latent variables are in ovals; their indicators are in rectangles; covariances are along the curved arrows; and error terms are e1 through $\mathrm{g} 9$. 


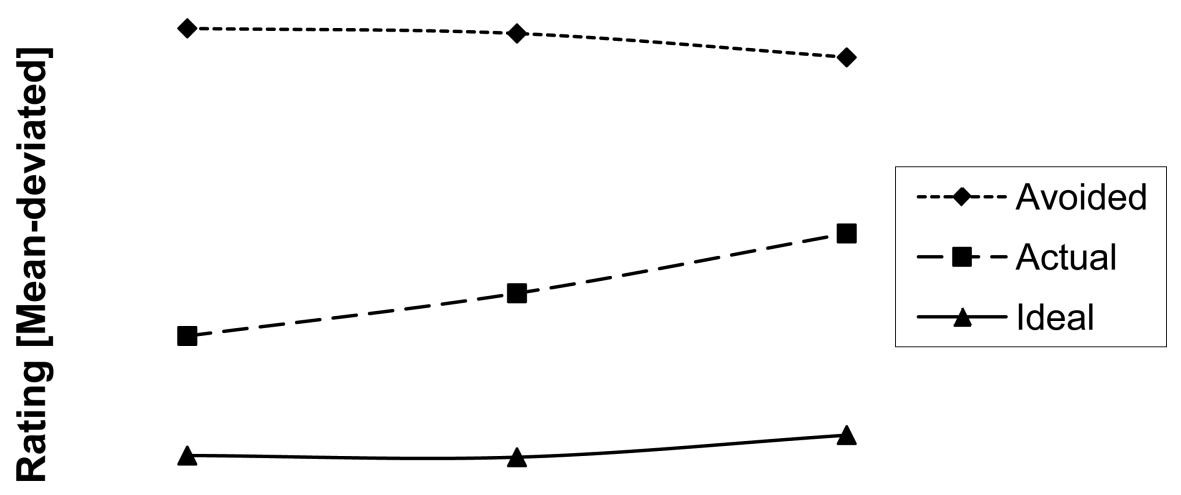

(a) European Americans

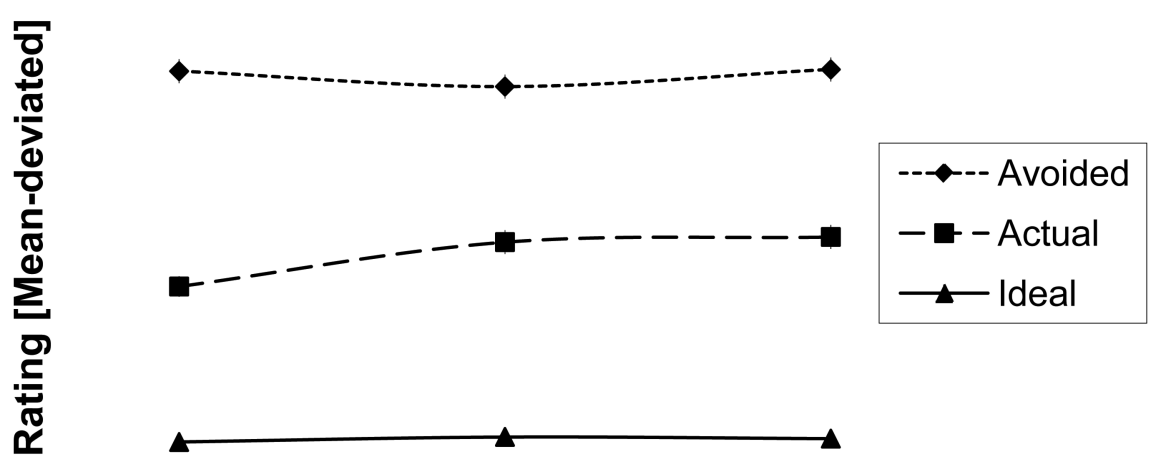

(b) Germans

3. Mean-deviated ratings of actual, ideal, and avoided affect in Study 2 for (a) European Americans and (b) Germans. HAN = high-arousal negative; $\mathrm{N}=$ negative; LAN = lowarousal negative. 


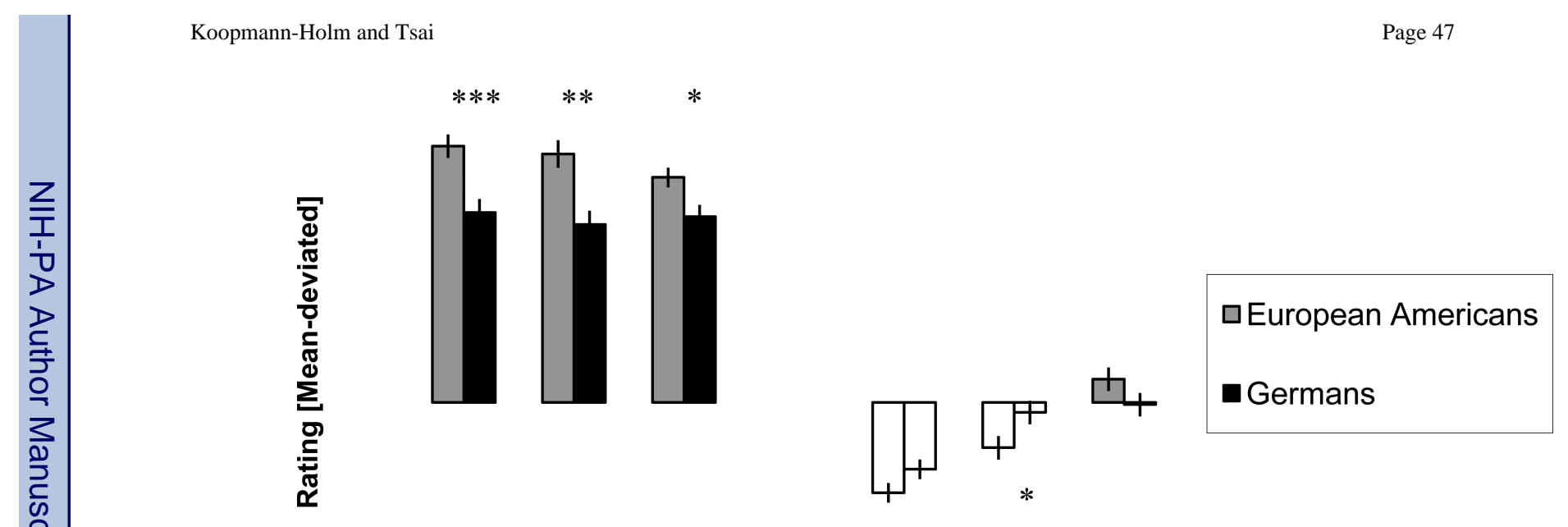

Avoided

Actual

4.

Group differences in avoided and actual negative affect in Study 2 (HAN = high-arousal negative; $\mathrm{N}=$ negative; $\mathrm{LAN}=$ low-arousal negative), controlling for actual and avoided negative affect, respectively. $* p<.05 ; * * p<.01 ; * * *<.001$. 
$* *$
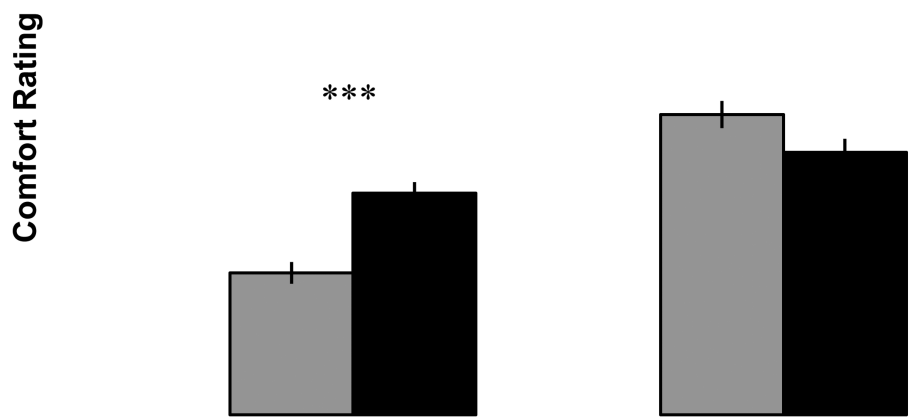

口European Americans

- Germans

(a)
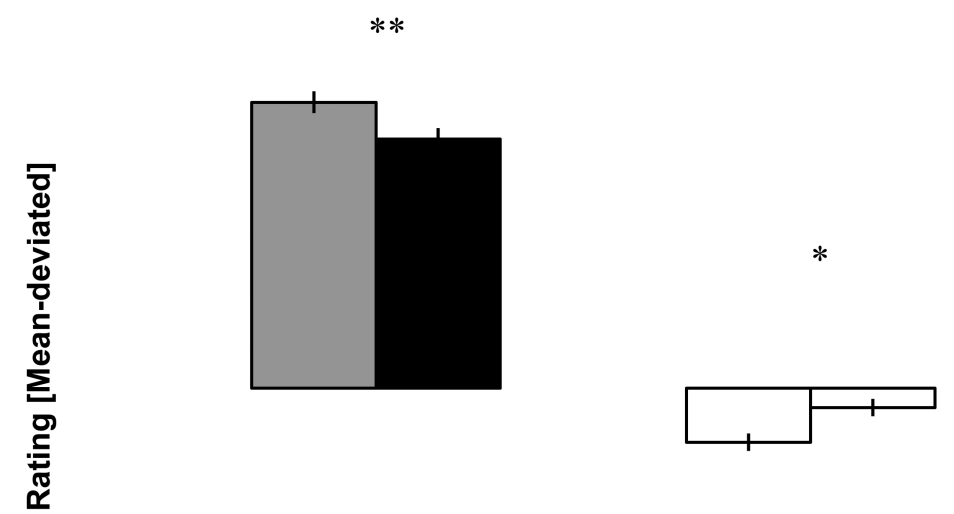

\section{口European Americans}

- Germans

(b)

5.

Group differences in (a) the comfort ratings of the negative and positive cards and (b) in avoided and actual negative affect in Study 3.

J Pers Soc Psychol. Author manuscript; available in PMC 2015 December 01. 


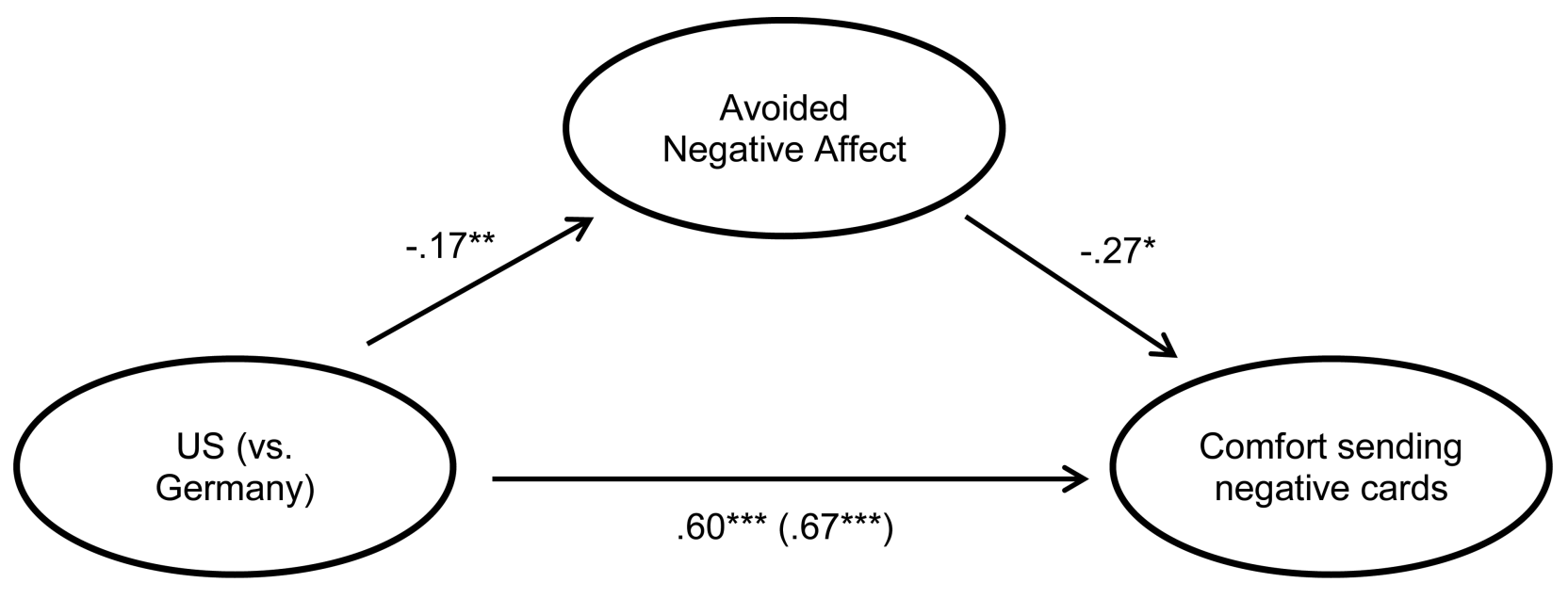

6.

Mediational model in Study 3 

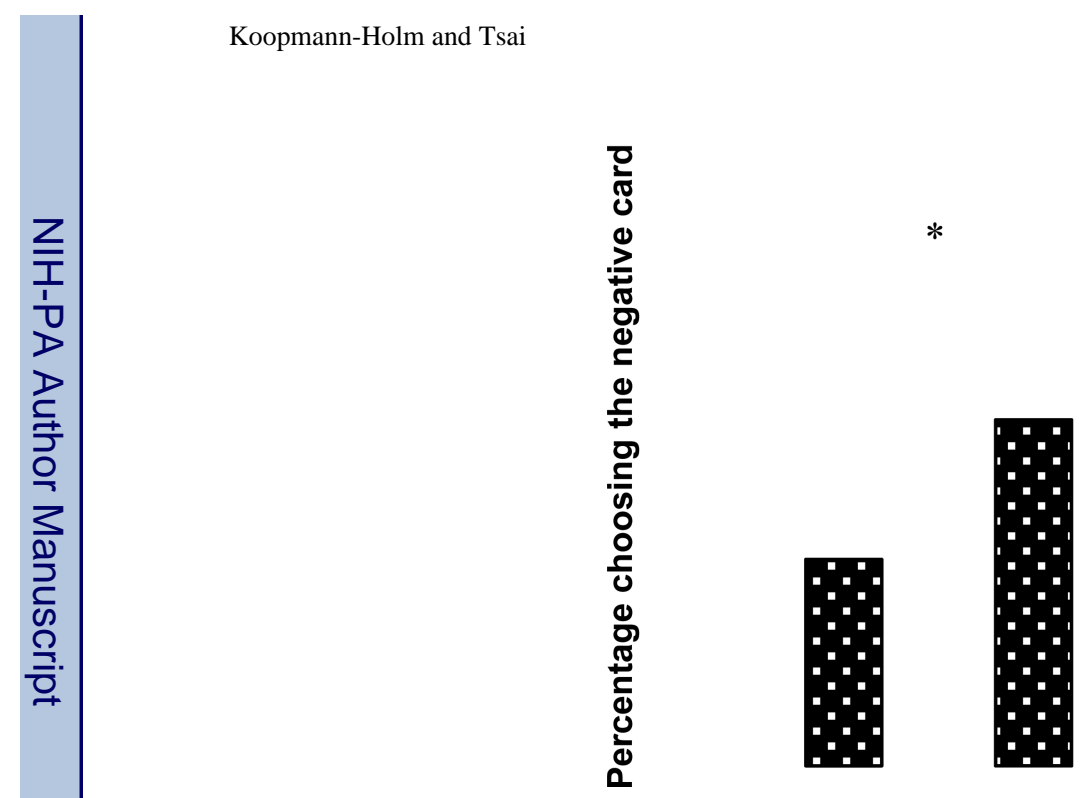

7.

Effect of Condition and Group on sympathy choice in Study $4 \mathrm{~b}$. 
Table 1

Zero-order correlations between the variables from the regressions (Study 3)

\begin{tabular}{|l|l|l|l|l|l|}
\hline & Country & Avoided negative affect & Actual negative affect & $\begin{array}{l}\text { Comfort rating of } \\
\text { negative cards }\end{array}$ & $\begin{array}{l}\text { Comfort rating } \\
\text { of positive } \\
\text { cards }\end{array}$ \\
\hline Country & & & & & \\
\hline Avoided negative affect & $-.19^{*}$ & & & & \\
\hline Actual negative affect & $.19^{*}$ & .09 & & & \\
\hline Comfort rating of negative cards & $.36^{* *}$ & $-.19^{*}$ & .11 & & \\
\hline Comfort rating of positive cards & $-.15^{*}$ & .02 & $-.19^{* *}$ & $.25^{* *}$ & \\
\hline
\end{tabular}

* $p<.05$

$* * \quad p<.01$ 